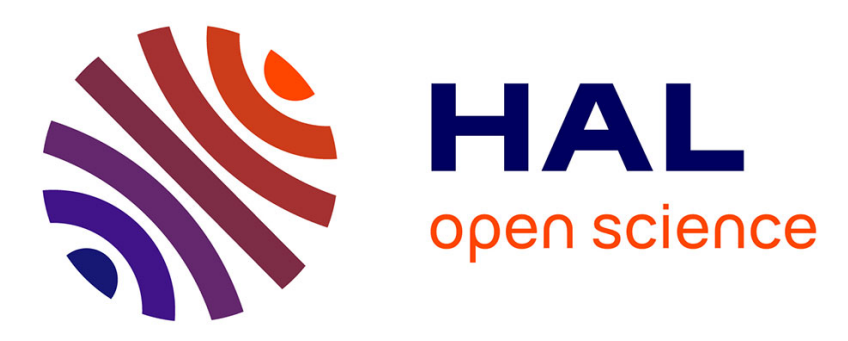

\title{
Valorisation économique des effets de la pollution atmosphérique
}

Olivier Chanel, Elsa Faugere, Ghislain Geniaux, Robert Kast, Stephane Luchini, Pascale Scapecchi

\section{- To cite this version:}

Olivier Chanel, Elsa Faugere, Ghislain Geniaux, Robert Kast, Stephane Luchini, et al.. Valorisation économique des effets de la pollution atmosphérique. Revue Economique, 2004, 55 (1), pp.65 - 92. 10.3917/reco.551.0065 . halshs-01664618

\section{HAL Id: halshs-01664618 https://shs.hal.science/halshs-01664618}

Submitted on 15 Dec 2017

HAL is a multi-disciplinary open access archive for the deposit and dissemination of scientific research documents, whether they are published or not. The documents may come from teaching and research institutions in France or abroad, or from public or private research centers.
L'archive ouverte pluridisciplinaire HAL, est destinée au dépôt et à la diffusion de documents scientifiques de niveau recherche, publiés ou non, émanant des établissements d'enseignement et de recherche français ou étrangers, des laboratoires publics ou privés. 


\section{VALORISATION ÉCONOMIQUE DES EFFETS DE LA POLLUTION ATMOSPHÉRIQUE}

Olivier Chanel, Elsa Faugère, Ghislain Geniaux, Robert Kast, Stéphane Luchini, Pascale Scapecchi

Presses de Sciences Po (P.F.N.S.P.) | « Revue économique »

2004/1 Vol. 55 | pages 65 à 92

ISSN 0035-2764

ISBN 2724629779

Article disponible en ligne à l'adresse :

https://www.cairn.info/revue-economique-2004-1-page-65.htm

\section{Pour citer cet article :}

Olivier Chanel et al., «Valorisation économique des effets de la pollution atmosphérique », Revue économique 2004/1 (Vol. 55), p. 65-92.

DOI 10.3917/reco.551.0065

Distribution électronique Cairn.info pour Presses de Sciences Po (P.F.N.S.P.).

(C) Presses de Sciences Po (P.F.N.S.P.). Tous droits réservés pour tous pays.

La reproduction ou représentation de cet article, notamment par photocopie, n'est autorisée que dans les limites des conditions générales d'utilisation du site ou, le cas échéant, des conditions générales de la licence souscrite par votre établissement. Toute autre reproduction ou représentation, en tout ou partie, sous quelque forme et de quelque manière que ce soit, est interdite sauf accord préalable et écrit de l'éditeur, en dehors des cas prévus par la législation en vigueur en France. Il est précisé que son stockage dans une base de données est également interdit. 


\title{
Valorisation économique des effets de la pollution atmosphérique
}

Presses de Sc. Po. | Revue économique

\author{
2004/1 - Vol. 55 \\ pages 65 à 92 \\ ISSN 0035-2764
}

Article disponible en ligne à l'adresse:

http://www.cairn.info/revue-economique-2004-1-page-65.htm

\section{Pour citer cet article :}

"Valorisation économique des effets de la pollution atmosphérique", Revue économique, 2004/1 Vol. 55, p. 65-92.

Distribution électronique Cairn.info pour Presses de Sc. Po..

(c) Presses de Sc. Po.. Tous droits réservés pour tous pays.

La reproduction ou représentation de cet article, notamment par photocopie, n'est autorisée que dans les limites des conditions générales d'utilisation du site ou, le cas échéant, des conditions générales de la licence souscrite par votre établissement. Toute autre reproduction ou représentation, en tout ou partie, sous quelque forme et de quelque manière que ce soit, est interdite sauf accord préalable et écrit de l'éditeur, en dehors des cas prévus par la législation en vigueur en France. II est précisé que son stockage dans une base de données est également interdit. 


\title{
Valorisation économique des effets de la pollution atmosphérique
}

\author{
Résultats d'une enquête contextuelle
}

\author{
Olivier Chanel* \\ Elsa Faugère** \\ Ghislain Geniaux** \\ Robert Kast* \\ Stéphane Luchini* \\ Pascale Scapecchi***
}

\begin{abstract}
Cet article présente les résultats d'une enquête d'évaluation contingente spécifique aux risques liés à la pollution de l'air (contextuelle) et estime les consentements à payer individuels pour les effets sanitaires (morbidité et mortalité) et non sanitaires. L'utilisation d'un scénario original auprès de 1273 habitants des Bouches-du-Rhône et un traitement économétrique adapté (modèle Box-Cox avec censure) conduisent à une valeur estimée de 68,60 euros par mois et par ménage pour une réduction de moitié du nombre de jours pollués, tous effets confondus. Sur la base d'un modèle d'utilité avec durée de vie aléatoire, nous évaluons pour la première fois une valeur d'évitement d'un décès dans le contexte de la pollution atmosphérique. Elle s'établit à 0,8 million d'euros.
\end{abstract}

\section{ECONOMIC VALUATION OF AIR POLLUTION EFFECTS : EVIDENCE FROM A CONTEXTUAL SURVEY}

This article introduces the main results of a contextual contingent valuation survey (i.e. specific to the underlying risk) dealing with a change in air pollution exposition. Individual willingness-to-pay for both health (morbidity and mortality) and non-health effects are elicited. The use of an original hypothetical scenario that involves 1273 inhabitants of the Bouches-du-Rhône and a convenient econometric model (Box-Cox model with censoring) lead to an overall predicted monthly value

* GREQAM-IDEP-CNRS, Marseille.

** INRA-SAD, Avignon.

*** OCDE, Paris.

Contact : Olivier Chanel, GREQAM-IDEP, Centre de la Vieille-Charité, 2, rue de la Charité, 13002 Marseille. Courriel : chanel@ehess.univ-mrs.fr

Les auteurs tiennent à remercier Dominique Ami, Jean-Jacques Becker, Yves Doazan et les deux rapporteurs anonymes pour leurs remarques et commentaires, les participants au séminaire Fourgeaud ainsi que le personnel de l'hôtel de région Provence-Alpes-Côte-d'Azur (PACA) dans lequel se sont déroulées les enquêtes simultanées. L'étude sous-tendant cet article a été financée par la Direction de la Prévention des pollutions et des risques du ministère de l'Écologie et du Développement durable (convention $n^{\circ} 36 / 98$ ) dans le cadre du programme PRIMEQUAL. 
of 68,6 euros per household for an half decrease of the number of polluted days. $A$ model of expected life-time resource allocation allows us to evaluate, for the first time, a value of a prevented fatality specific to the air pollution risk: 0,8 million Euros.

Classification JEL : C1, I1, Q25, D12

\section{INTRODUCTION}

Les préoccupations croissantes de la population pour l'environnement conduisent les pouvoirs publics à engager des politiques de préservation et de restauration du patrimoine naturel et à imposer, pour tout projet susceptible de porter atteinte à l'environnement, une étude préalable des impacts. La loi sur l'eau initiée en janvier 1992, puis renforcée en 2001, et plus encore celle sur l'air de décembre 1996 témoignent de l'importance qu'occupent actuellement les questions d'environnement dans l'action publique. Toutefois, les coûts élevés des politiques environnementales et les besoins vitaux d'autres domaines de l'action publique, comme l'emploi, la santé ou l'éducation, rappellent que les préoccupations en matière d'environnement s'intègrent dans un contexte d'arbitrage, régi par des contraintes budgétaires. Une solution à ce problème de choix public consiste à valoriser monétairement les bénéfices associés à une politique environnementale, afin de les comparer aux coûts de sa mise en ouvre, et de déterminer, sur une base économique, les décisions les plus bénéfiques pour la collectivité. Ainsi en est-il de l'amélioration de la qualité des nappes phréatiques, de la préservation des sites naturels ou de la lutte contre la pollution atmosphérique.

La pollution atmosphérique est susceptible d'engendrer trois types d'effets sur le bien-être : des effets non sanitaires directs - dégradation des perceptions sensorielles - et indirects - altération de la flore, de la faune, des cultures ou des bâtiments - et des effets sanitaires directs - mortalité et morbidité (hospitalisations et maladies). Une politique de réduction des émissions génère donc différents types de bénéfices que des méthodes adaptées permettent d'évaluer. Si l'on se focalise sur les bénéfices sanitaires relatifs à une réduction de la pollution, le recours à trois méthodes de valorisation est envisageable.

1. La première repose sur l'observation des valeurs économiques associées à des épisodes morbides ou à des pertes de production induites par un décès. Appelée méthode du coût marchand (MCM), elle valorise les épisodes morbides à partir des prix que le marché attribue, à l'équilibre, à leur traitement sanitaire (prix d'une hospitalisation, d'une consultation, des soins...). Cette méthode suppose implicitement que les caractéristiques de ces épisodes morbides ne diffèrent pas de celles de mêmes épisodes attribuables à d'autres causes. Appelée méthode des pertes de production (MPP) pour la mortalité, ou improprement méthode du capital humain, elle fonde la valeur sur l'activité productive de l'individu, évaluée comme la somme actualisée des revenus futurs pour chaque âge (Landefeld et Seskin [1982]). Cette approche est largement critiquée puisque, outre le fait que le choix du taux d'actualisation constitue une variable clef, elle repose sur un ensemble d'hypothèses discutables: la valeur de l'individu est représentée 
uniquement par ce qu'il produit ; cette productivité est correctement et uniquement mesurée par les revenus du travail ; elle ne tient pas totalement compte des préférences des agents dont toute valeur économique est pourtant issue. La fragilité de la MPP explique qu'elle soit tombée en relative désuétude.

2. La seconde méthode consiste à fonder le calcul des bénéfices sur les préférences des individus en utilisant l'information disponible sur divers marchés : marché du travail, du logement ou des biens de protection. Elle est qualifiée de méthode de révélation indirecte des préférences puisqu'elle conduit à une valorisation ex post en estimant la contrepartie monétaire nécessaire pour accepter une variation du niveau de risque (professionnel, lié à l'habitat ou aux biens de protection) en s'appuyant sur des propriétés de substituabilité ou de complémentarité entre des biens marchands et les risques de morbidité ou de mortalité que l'on cherche à estimer. L'utilisation de cette méthode est cependant conditionnée par le fait qu'il soit possible d'observer des liens entre des comportements d'achat sur des marchés réels et différentes configurations des niveaux de pollution de l'air, et d'identifier la fonction de demande en qualité de l'air. Au-delà de la difficulté d'observer systématiquement ces comportements, leur association exclusive à des préférences en matière de pollution de l'air est difficile et, par là même, discutable (voir, par exemple, Palmquist [1991], pour un exposé complet).

3. Confrontés à ces difficultés, les économistes mobilisent, de manière croissante, une troisième méthode : la méthode d'évaluation contingente (MEC). Elle permet de déterminer ex ante l'équivalent monétaire d'une variation de bien-être des individus par le biais d'enquêtes utilisant des scénarios hypothétiques. Tout comme pour les deux méthodes précédentes, il peut s'agir de consentements à payer (CAP) spécifiques à différentes composantes de leur santé. Nous avons alors affaire à une approche chaînée, qui nécessite la combinaison de données, de la variation des concentrations jusqu'aux impacts en termes de mortalité ou de morbidité, afin d'obtenir une valorisation des effets associés à une réduction de la pollution. Toutefois, la MEC permet également de valoriser directement l'ensemble des effets, y compris les effets ne possédant pas de prix per se (peine, souffrance, temps perdu, ...), en évaluant les CAP pour une variation de la qualité de l'air en général. Il s'agit alors d'une approche intégrée, plus conforme à l'économie du bien-être, puisqu'il revient aux individus d'estimer eux-mêmes, sur la base de l'information communiquée dans le scénario, l'impact de la modification du niveau de pollution atmosphérique sur leur bien-être. Les CAP obtenus peuvent alors largement dépendre des perceptions, représentations et habitudes individuelles propres à la pollution de l'air et aux risques éventuels qu'elle génère. Si tel est le cas, la transférabilité des valeurs d'une situation à une autre s'avère très discutable et impose la nécessité de valeurs monétaires contextuelles, c'est-à-dire de valeurs monétaires établies sur des scénarios spécifiques au problème étudié.

Au-delà du choix d'une méthode particulière, il est nécessaire de valoriser de façon monétaire les conséquences d'une amélioration de la pollution de l'air en termes de mortalité afin de garantir l'efficacité économique de toute décision collective susceptible de modifier la probabilité de décès. Proposer une valeur d'évitement d'un décès (VED) contextuelle, fondée sur une théorie économique, constitue un enjeu de décision publique puisque la santé représente la majorité des bénéfices à attendre d'une politique de réduction de la pollution atmosphérique (autour de $80 \%$ en Europe par exemple pour les Nox et les composés orga- 
niques volatils d'après Holland et King [1998]). À elle seule, la mortalité (en particulier associée à une exposition de long terme) représente entre $75 \%$ et $95 \%$ de l'ensemble des effets sanitaires (Holland et King [1998, 1999], Künzli et al. [2000], Chanel et al. [1996]).

Toutefois, bien que plusieurs recherches soient en cours sur ce thème, une seule VED adaptée à une cause environnementale est jusqu'à présent disponible (Krupnick et al. [2002]). Elle est établie sur la base d'une évaluation contingente et conduit à une valeur moyenne de 0,5 million d'euros 1999 pour un décès associé à la pollution atmosphérique. Elle ne peut néanmoins être considérée comme contextuelle car elle ne repose pas sur un scénario spécifique : seule la tranche d'âge (plus de 70 ans) à laquelle elle est calculée correspond aux âges auxquels les décès consécutifs à une exposition surviennent. Lorsque l'évaluation repose sur un contexte différent, on corrige généralement (arbitrairement) son influence ex post : UK DH [1999] ou Sommer et al. [1999] proposent par exemple de doubler ou de tripler la valeur obtenue pour une cause de décès « accident de la route » pour tenir compte de la différence entre les risques sousjacents.

À la différence des travaux entrepris jusqu'à présent pour évaluer les effets de la pollution atmosphérique sur le bien-être, nous proposons, dans cet article, une évaluation monétaire contextuelle des impacts de la pollution de l'air à partir d'une enquête d'évaluation contingente menée sur la population des Bouchesdu-Rhône. Dans la section 2, nous présentons un aperçu des biais généralement rencontrés dans la mise en œuvre de la MEC et commentons les plus préoccupants dans le contexte de la pollution atmosphérique. Cela nous conduit à définir les modalités d'un scénario hypothétique original que nous décrivons et justifions. Dans la section 3, nous évaluons, à l'aide d'un modèle économétrique spécifique, les CAP pour l'ensemble des effets de la pollution (morbidité, mortalité et effets non sanitaires) en tenant compte des différentes perceptions de la pollution de l'air. Dans la section 4, nous nous concentrons sur l'estimation d'une VED contextuelle, sur la base d'un modèle théorique adapté. Nous discutons enfin les résultats obtenus dans la conclusion.

\section{CHOIX D'UN SCÉNARIO HYPOTHÉTIQUE PERTINENT}

La méthode d'évaluation contingente consiste à évaluer, à l'aide de questions appropriées, combien les individus sont prêts à payer ex ante (leur consentement à payer, CAP) pour une modification donnée (quantitative ou qualitative) d'un bien environnemental. Les individus sont donc placés dans une situation hypothétique et les réponses obtenues dans les enquêtes correspondent à des intentions de paiement sur un marché tout aussi hypothétique. Ce dernier doit être rendu aussi crédible que possible au moyen d'un scénario décrivant l'ensemble des informations pertinentes, afin de garantir des comportements aussi proches que possible de ceux que l'on observerait sur un marché. Le scénario doit intégrer les caractéristiques du bien offert, les conditions dans lesquelles il sera mis à disposition ainsi que les modalités de prélèvement des CAP déclarés, le véhicule de paiement (prélèvements obligatoires, droits d'accès, etc.). On conçoit aisément que l'exercice est délicat puisque les montants révélés dépendront de la 
formulation du scénario, chacun de ses éléments constitutifs pouvant se révéler une source potentielle de biais. Dans cette section, nous reprenons, en premier lieu, les biais les plus souvent évoqués dans la littérature. Nous présentons ensuite le scénario retenu et montrons comment il minimise ces biais. La dernière partie de la section détaille la construction des questionnaires.

\section{Biais inhérents à la méthode}

La notion de biais à laquelle nous faisons référence ici suppose implicitement que chaque individu valorise le bien sur la base d'une maximisation d'utilité, conformément à la théorie du consommateur. Tout écart à ce programme d'optimisation est alors considéré comme un biais, c'est-à-dire comme une source d'erreur systématique non aléatoire à minimiser. Les biais potentiels de la méthode d'évaluation contingente ont été largement commentés dans la littérature (voir Mitchell et Carson [1989], pour une typologie ou l'ouvrage édité par Hausman [1993], pour une analyse critique de la MEC). Les recommandations du NOAA Panel (Arrow et al. [1993]) ainsi que des développements méthodologiques récents permettent de réduire l'importance des biais, voire d'éviter totalement certains d'entre eux, que ce soit lors du choix du scénario, du mode de questionnement, ou lors des traitements économétriques. Nous présentons ci-dessous deux sources majeures de biais liées aux comportements d'individus placés sur un marché hypothétique (biais stratégique et hypothétique) ainsi que le biais d'inclusion, plus directement lié à une mauvaise spécification du scénario ${ }^{1}$.

Le biais stratégique survient lorsque les individus pensent que leurs réponses peuvent influencer la décision finale d'accord ou de rejet de la politique décrite dans le scénario, et répondent en conséquence. Ce biais implique que les individus ont une représentation relativement claire des conséquences du projet, et qu'ils disposent d'informations sur les critères de choix qui vont jouer dans la décision (coût global du projet, nombre de personnes susceptibles d'être mis à contribution financière, répartition des charges).

Le biais hypothétique survient lorsque l'individu ne peut se projeter dans la situation d'une transaction hypothétique. Ce biais caractérise les écarts potentiels entre déclarations (réponses hypothétiques) et comportements réels. Par nature, le contexte hypothétique de la situation ne peut être éliminé ${ }^{2}$ et le manque de pertinence d'un scénario peut alors conduire à accroître systématiquement les écarts entre déclarations et comportements réels. Ce biais résulte généralement de trois types de faiblesses inhérentes aux évaluations contingentes, détaillées dans l'encadré 1 : le manque de familiarité avec le marché hypothétique, le manque d'information sur le bien évalué et l'incertitude.

Le biais d'inclusion (ou embedding effect; part-whole bias) se définit comme la tendance des individus à donner une valeur globale à un bien sans tenir compte

1. Nous renvoyons à Chanel et al. [2001] pour l'exposé des autres biais, notamment ceux inhérents aux modes d'élicitation des préférences (véhicules de paiement, format de réponses, biais d'ancrage, etc.)

2. C'est pour cette raison que certains auteurs préfèrent parler de contexte hypothétique plutôt que de biais hypothétique. 
de sa délimitation précise au sein de l'ensemble des biens dans lequel il s'insère (Boyle [1994]). Il implique que le bien, dans la description qui en est proposée, est perçu de façon erronée par les individus. L'importance de ce biais dépend de la nature des biens que l'on veut évaluer : plus les individus ont des préférences identifiées et marquées pour les biens considérés - c'est notamment le cas pour les biens publics très localisés et lorsqu'il est question de valeur d'usage - moins ce biais se révèle important.

\section{Encadré 1}

\section{PROBLÈMES INDUITS PAR LE CONTEXTE HYPOTHÉTIQUE DE L'ÉVALUATION}

\section{La familiarité avec la question posée}

Les individus n'ont pas de préférences, au sens économique du terme, clairement définies pour des biens avec lesquels ils n'ont pas d'expérience directe d'usage ou d'échange, comme les biens non marchands ou les actifs environnementaux. Pour Diamond et Hausman [1993], la non-familiarité est double puisqu'elle concerne le bien et le marché hypothétique associé. La MEC étant une procédure de recueil d'opinions, il importe que ces opinions soient définies sur le bien évalué, ce qui implique un certain niveau de familiarité des individus avec ce bien (usages directs ou indirects fréquents, connaissances de ces usages, questionnements autour de ce bien dans les débats locaux). La question de la familiarité au marché contingent est moins cruciale puisqu'elle se résume à l'utilisation d'un véhicule de paiement habituel et adapté.

\section{Le niveau d'information}

L'information délivrée au cours du scénario est susceptible d'influencer la révélation de la valeur lorsque des variations de risque de disparition d'un bien environnemental sont évaluées. Le problème de la connaissance ou de la non-connaissance de ce risque crée une difficulté supplémentaire puisque certains individus apprennent simultanément l'existence et la menace qui pèsent sur le bien environnemental. Ensuite, familiarité et niveau d'information sont intrinsèquement liés : plus l'individu est familier avec le bien, plus l'information dont il dispose au préalable est importante, et moins son CAP exprimé sera influencé. Les études menées sur l'effet de l'information dans une évaluation contingente montrent un effet positif sur les réponses quand les individus n'ont pas le même ensemble informationnel initial (Blomquist et Whitehead [1991]), ainsi que l'existence d'un seuil informatif à partir duquel l'ajout d'information ne modifie plus les décisions individuelles (Hanley et Munro [1992]).

\section{L’incertitude produite par le scénario}

Les individus interrogés sont confrontés à une double incertitude : l'une relative à l'offre (le bien hypothétique, généralement un projet d'action publique) et l'autre à la demande (les préférences des individus au moment de la mise en œuvre du projet). Ces incertitudes provoquent une forte sensibilité des réponses à la façon dont les questions sont posées (framing effect pour reprendre la terminologie de Tversky et Kahneman [1981]), à l'information de fond qui est délivrée, à l'ordre des questions, etc. La nature hypothétique du scénario peut ainsi impliquer un biais de surengagement (les achats sur un marché réel nécessitent une dépense réelle, ce qui n'est pas le cas de réponses à une enquête ; voir Kemp et Maxwell [1993], Mitchell et Carson [1989]) dès lors que le bien est perçu positivement. La solution la plus évidente est la troncature à droite de l'échantillon ou l'utilisation d'une transformation de type Box-Cox afin de normaliser la distribution des erreurs. 


\section{Scénario et contrôle des biais}

Le choix méthodologique central a porté sur la nature du scénario le plus susceptible de contrôler les biais présentés précédemment. Le scénario hypothétique retenu place l'individu face à l'obligation de déménager avec sa famille ${ }^{1}$. Deux localisations lui sont proposées, similaires en tous points (taille de la ville, logement, climat, équipements sportifs, vie culturelle, dessertes en transports...) à deux exceptions près : le niveau de pollution et le coût de la vie (voir annexe A pour l'énoncé exact du scénario).

Ce scénario se distingue fondamentalement des scénarios généralement utilisés dans la MEC. Dans ces derniers, l'évaluation porte sur les CAP maxima pour passer d'une situation de référence (généralement la situation actuelle) à une situation hypothétique dans laquelle l'action publique a permis la délivrance d'une offre de biens publics différente (amélioration de la qualité d'une ressource naturelle, réduction d'une nuisance, préservation d'un site ou d'une espèce...). Ne pas appuyer le scénario sur une action publique mais sur une privatisation du bien « pollution de l'air » possède de nombreuses vertus.

D'abord, l'offre est certaine. Ainsi, les biais imputables à l'incertitude sur la nature du bien offert dans le marché contingent sont minimisés et seuls demeurent des problèmes de perception individuelle du bien proposé (voir annexe $\mathrm{B}$ ). La délimitation de la variation de qualité environnementale est dès lors plus précisément perçue, ce qui réduit notablement les effets possibles du biais d'inclusion.

Ensuite, le choix proposé aux individus renvoie à un espace de choix strictement individuel (restreint au cadre du ménage) et sans effet direct sur la décision publique $^{2}$. On attend de cette situation de choix plusieurs propriétés. D'abord, elle invalide tout biais stratégique puisque aucune décision publique n'est en jeu dans le scénario proposé : les deux situations à comparer existent déjà et toute spéculation sur l'utilisation des réponses fournies devient un exercice trop complexe pour que les individus y aient systématiquement recours. Ensuite, cela permet de réduire plus encore le biais d'inclusion puisque cette absence de liens entre CAP révélé et action publique à venir permet également que ne soient pas révélés indûment des CAP pour l'environnement en général ou dépassant le cadre strict des effets sanitaires de la pollution de l'air. La transaction proposée affiche un gain certain en termes d'effets sanitaires de la pollution de l'air et uniquement cela, et le véhicule de paiement utilisé (un coût additionnel au coût de la vie) ne permet aucun usage public de ces fonds. Enfin, cette situation de choix évite les réponses altruistes ou citoyennes qui invalident généralement la construction d'une préférence collective sur la base de choix individuels (Sagoff [1998]; Common, Reid et Blamey [1997]).

Enfin, concernant la familiarité avec le marché hypothétique, celui que nous proposons place les individus dans une situation très proche de celle qu'ils

1. Jones-Lee et al. [1999] procèdent de manière comparable pour étudier les consentements à payer pour réduire le risque d'accident de la route en Nouvelle-Zélande.

2. Le scénario retenu (déménagement de l'enquêté et des membres composant sa famille) n'affecte pas le niveau de pollution observé dans les deux lieux de résidence. Le CAP de l'individu pour vivre dans un environnement moins pollué représente donc une dépense personnelle prélevée sur son budget qui n'a aucune raison d'influencer la mise en place d'une politique. 
rencontrent dans la vie courante. En effet, les dimensions privée et économique sont centrales dans les décisions de localisation des ménages, et ce type de décision est plus étroitement lié avec la sphère marchande que ne peuvent l'être des scénarios proposant des améliorations de la qualité environnementale via des actions publiques. Par ailleurs, même si d'autres critères que la pollution atmosphérique s'avèrent déterminants dans les choix de localisation effectifs, le scénario retenu en imposant une similitude des deux villes, excepté sur les deux critères qui nous intéressent (pollution et coût de la vie), et en s'appuyant sur deux villes abstraites, permet de rendre pertinents les arbitrages sous-jacents. L'incertitude ne repose alors que sur l'enquêté qui doit décider de son lieu de résidence. Quant à l'effet du véhicule de paiement, le fait de le présenter comme un coût supplémentaire venant s'ajouter aux dépenses mensuelles courantes est susceptible de réduire l'écart entre discours et pratique (biais hypothétique). Cela limite considérablement le risque de réponses de protestation que sont susceptibles de provoquer d'autres modes de paiement (taxe, impôt ou autre). Enfin, une somme mensuelle par ménage est a priori proche du cadre de raisonnement des individus : loyer, remboursement de prêts, abonnement de transport, mensualisation des taxes ou des factures, revenus salariés sont en effet généralement mensuels.

\section{Construction des questionnaires}

L'essentiel de nos préoccupations, dans la procédure de calibrage du questionnaire, s'est centré autour de trois exigences : concevoir un mode de description du scénario permettant d'être compris et perçu par les individus interrogés ; disposer des moyens d'étudier l'effet d'un accroissement de l'information délivrée sur les liens entre pollution de l'air et effets sanitaires ; recueillir des renseignements sur l'ensemble des pratiques et des représentations individuelles susceptibles d'être déterminantes dans la perception des risques sanitaires de la pollution de l'air' ${ }^{1}$. Dans cette perspective, trois étapes ont été nécessaires pour élaborer le questionnaire définitif : enquête anthropologique, conception et pré-tests des questionnaires initiaux, élaboration et mise en œuvre des questionnaires finals.

Deux enquêtes à dominante anthropologique ont été réalisées par entretiens semi-directifs individuels et collectifs en 1999 et en 2000 (73 personnes), certains ayant recours au support photographique. Au-delà de ses objectifs propres, l'analyse du contenu de ces entretiens a fourni des bases à la construction du questionnaire d'évaluation proprement dit, en facilitant l'utilisation d'un vocabulaire et la construction d'un scénario adaptés, par l'insertion de questions spécifiques aux représentations individuelles de la pollution de l'air, et par la mise en évidence des difficultés liées à la monétarisation d'une variation de risque liée à la pollution de l'air.

1. Toute enquête d'évaluation comprend un volet collectant les informations propres à l'enquêté, et un volet dans lequel est exposé le scénario hypothétique. Outre les caractéristiques socio-démographiques habituelles (29 questions), les thèmes santé, pratiques alimentaires et sport (15 questions), comportements vis-à-vis des risques (4 questions) et environnement (16 questions) ont été particulièrement renseignés, en particulier par neuf thèmes que l'analyse anthropologique préalablement menée a jugés susceptibles d'être discriminants en matière de comportement vis-à-vis de ce type de risque. 
Le pré-test s'est effectué sur la base d'une première version du questionnaire, administrée en face à face à une vingtaine de personnes, à laquelle succédait une discussion ouverte sur les problèmes rencontrés par l'enquêté. La pré-enquête s'est ensuite déroulée sur la base d'une version quasi définitive du scénario et en condition réelle (dans l'hémicycle de l'hôtel de région PACA). Elle a permis des modifications mineures du questionnaire, et l'obtention de valeurs monétaires qui ont servi d'offres de départ dans le processus à deux offres successives utilisé lors des enquêtes téléphoniques.

Concrètement, les scénarios retenus in fine ont pour caractéristiques communes une situation de choix individuel entre deux localisations: la première possède un niveau de pollution moyen correspondant à celui observé dans Marseille, et la seconde propose un niveau de pollution plus faible (« deux fois moins de jours de pollution ») mais associé à un coût de la vie plus élevé. Choisir une seule localité de référence était nécessaire pour disposer d'une référence commune à tous les individus, que ce soit en termes de perceptions de la pollution, que, de façon plus formelle, de niveau d'utilité. Puisque la majorité des répondants $(54 \%)$ résident à Marseille et que les $46 \%$ restants sont vraisemblablement déjà venus à Marseille pour des raisons administratives, professionnelles ou personnelles, cette ville constitue une référence naturelle. Le concept de «deux fois moins de jours de pollution » a été préféré à une référence à des concentrations dans la mesure où les entretiens semi-directifs préalables ont montré qu'il était plus familier aux individus (Faugère [2002]). Il est progressivement explicité au cours du déroulement de l'enquête afin d'étudier l'impact d'une révélation d'information sur les consentements à payer (voir annexe A). Le consentement à payer pour une diminution de la pollution atmosphérique en général est d'abord recherché (CAP sans information), avant de préciser les impacts qu'aurait une telle diminution (CAP avec information) en termes d'effets non sanitaires, de réduction des épisodes morbides et de réduction de la mortalité. La réduction de la mortalité est l'objet d'une valorisation spécifique sur une partie de l'échantillon afin de permettre le calcul d'une VED contextuelle (voir supra).

Un échantillon de 1273 habitants des Bouches-du-Rhône a été enquêté, en juin 2000 et en juin 2001, selon deux modes d'administration. Un format classique par voie téléphonique, d'une durée moyenne d'une vingtaine de minutes, a porté sur deux échantillons de 503 personnes, représentatifs de la population des Bouches-du-Rhône ${ }^{1}$. Un format moins conventionnel a concerné deux échantillons de 142 et de 125 personnes regroupées dans un même lieu (l'hémicycle de l'hôtel de région PACA), qui ont répondu aux questions de révélation par vote électronique lors de deux séances d'une durée moyenne d'une heure ${ }^{2}$.

1. Du fait de contraintes budgétaires, la phase téléphonique a dû être effectuée en deux échantillons. La sélection des deux échantillons téléphoniques s'est fondée sur la méthode des quotas. La stratification comportait quatre classes, sur la base des données INSEE 1990 : le sexe, l'âge (18-29 ans, 30-59 et 60 et plus), le lieu de résidence (moins de 20000 habitants, 20-100 000, 100-250 000 et plus de 250000 ) et les catégories socio-professionnelles (travailleurs indépendants, cadres et professions intermédiaires, employés et ouvriers, non-actifs). Chacun des deux échantillons est représentatif de la population des Bouches-du-Rhône.

2. Ces deux échantillons comportent plus de jeunes, moins d'actifs (une proportion plus importante d'étudiants et de chômeurs), plus d'urbains et légèrement plus de femmes que la population des Bouches-du-Rhône et n'en sont donc pas représentatifs. 
Le mode d'élicitation, c'est-à-dire la façon dont les questions portant sur l'évaluation monétaire sont posées, est susceptible d'introduire un biais fréquent dans les enquêtes d'évaluation contingente : le biais d'ancrage ou biais du point de départ (Mitchell et Carson [1989] ; Herriges et Shogren [1996], Green et al. [1998]). En effet, les questions fermées présentent un inconvénient: elles peuvent laisser supposer à la personne interrogée que le montant proposé est proche de la vraie valeur, si bien que cette dernière peut ancrer son CAP sur l'offre de départ proposée, rendant le résultat sensible aux niveaux des offres proposées. L'avantage de ce type de format est cependant de permettre de réduire le taux de non-réponse, les valeurs nulles et extrêmes, difficiles à contrôler. Nous avons choisi un mode mixte : la personne interrogée a d'abord répondu à une succession de questions fermées ascendantes pour l'enquête simultanée et à un mécanisme comportant deux offres successives pour l'enquête téléphonique, avant d'indiquer, pour les deux types de questionnement, un montant monétaire sur la base d'une question ouverte. Procéder ainsi fournit plus d'information sur les CAP mais nécessite la prise en compte de l'ancrage des réponses sur la valeur de départ dans le traitement économétrique.

\section{CONSENTEMENTS À PAYER POUR UNE DIMINUTION DE LA POLLUTION DE L'AIR}

\section{Modèle économétrique}

La recherche d'une meilleure compréhension des déterminants des CAP pour une amélioration de la qualité de l'air nécessite la mise en œuvre d'un modèle adapté. Toutefois, il n'est pas possible de se référer sans ambiguïté à un modèle d'utilité aléatoire conventionnel puisque la pollution de l'air met en jeu des risques. Ce n'est donc plus la théorie du consommateur dans un univers certain qui est en jeu mais bien une théorie de la décision face au risque ${ }^{1}$. Il n'existe cependant pas, dans la littérature en évaluation contingente, de modèles économétriques structurels incorporant la dimension risque. Nous retenons ici un modèle dérivé de McFadden et Leonard [1993] pour sa grande flexibilité. Formellement, nous avons pour l'individu $i$ :

$$
\mathrm{G}\left(\mathrm{CAP}_{i}, y_{i} ; \alpha\right)=\mathrm{Z}\left(\mathrm{X}_{i}, \epsilon_{i} ; \gamma\right)
$$

avec $\mathrm{CAP}_{i}$ le consentement à payer de l'individu $i, y_{i}$ son revenu, $\mathrm{X}_{i}$ l'ensemble des variables explicatives, $\theta=(\alpha, \gamma)$ un vecteur de paramètres et $\epsilon_{i}$ est le terme d'erreur associé à l'individu $i$. La fonction $\mathrm{G}($.$) est définie$ comme :

$$
\mathrm{G}\left(\mathrm{CAP}_{i}, y_{i} ; \alpha\right)= \begin{cases}\frac{y^{1-\alpha}-\left(y-\mathrm{CAP}_{i}\right)^{1-\alpha}}{1-\alpha} & \text { si } \alpha \neq 1 \\ -\log \left(1-\mathrm{CAP}_{i} / y_{i}\right) & \text { si } \alpha=1\end{cases}
$$

1. Le consentement à payer n'est donc pas un surplus du consommateur mais un prix d'option, par exemple. 
Cette transformation Box-Cox de la variable dépendante permet une grande flexibilité dans la relation entre le revenu et le consentement à payer puisqu'elle recouvre plusieurs des modèles utilisés dans la littérature : lorsque $\alpha=0$, le modèle économétrique devient un simple modèle linéaire et lorsque $\alpha=1$, le modèle économétrique est un modèle logarithmique. Dans le cadre d'un modèle d'utilité aléatoire, le paramètre $\alpha$ peut s'interpréter comme l'élasticité du consentement à payer par rapport au revenu.

La fonction $z_{i}=\mathrm{Z}\left(\mathrm{X}_{i}, \epsilon_{i} ; \gamma\right)$ capture l'hétérogénéité individuelle sur la base des caractéristiques observables des individus et d'un terme d'erreur aléatoire $\epsilon_{i}$. Les composantes de $z$ varient donc dans la population selon une distribution induite par la distribution des termes d'erreur $\epsilon_{i}$.

On déduit aisément des équations (1) et (2) le consentement à payer $\mathrm{CAP}_{i}$ satisfaisant $z_{i}=\mathrm{G}\left(\mathrm{CAP}_{i}, y_{i} ; \alpha\right)$ comme :

$$
\mathrm{CAP}_{i}=y_{i}-\left[y_{i}^{1-\alpha}-(1-\alpha) z_{i}\right]^{1 /(1-\alpha)}
$$

La distribution $\mathrm{F}\left(z_{i} ; \gamma\right)$ des $z_{i}$ induit la fonction de distribution des consentements à payer dans la population comme

$$
\mathrm{F}_{\mathrm{CAP}}\left(\mathrm{CAP}_{i}, y_{i} ; \alpha, \gamma\right)=\mathrm{F}_{z}\left(\mathrm{G}\left(\mathrm{CAP}_{i}, y_{i} ; \alpha\right) ; \gamma\right) \text {. }
$$

Ainsi, si le consentement à payer de l'individu $i$ est strictement positif, $\mathrm{CAP}_{i}$ a pour densité :

$$
f_{\mathrm{CAP}}\left(\mathrm{CAP}_{i}, y_{i} ; \alpha, \gamma\right)=\frac{f_{z}\left(\mathrm{G}\left(\mathrm{CAP}_{i}, y_{i} ; \alpha\right) ; \gamma\right)}{\left(y_{i}-\mathrm{CAP}_{i}\right)^{1-\alpha}}
$$

Dans le cas où le consentement à payer observé est nul, l'observation $i$ est dite censurée à gauche. La contribution à la vraisemblance d'une observation censurée est alors définie sur la base de la fonction de répartition :

$$
\mathrm{F}_{z}\left(\mathrm{G}\left(0, y_{i} ; \alpha\right) ; \gamma\right)
$$

Si les termes d'erreurs sont indépendamment et identiquement distribués selon une loi normale d'espérance nulle et d'écart type $\sigma$, la contribution de l'observation $i$ à la fonction de log-vraisemblance s'écrit de la manière suivante :

$$
\ell_{i}=c_{i} \log \left[\Phi\left(\frac{\mathrm{X}_{i} \beta}{\sigma}\right)\right]+\left(1-c_{i}\right) \log \left[\frac{\phi\left(\left(\mathrm{G}\left(\mathrm{CAP}_{i}, y_{i} ; \alpha\right)-\mathrm{X}_{i} \beta\right) / \sigma\right)}{\sigma\left(y_{i}-\mathrm{CAP}_{i}\right)^{1-\alpha}}\right]
$$

où $c_{i}$ est une indicatrice prenant la valeur 1 si le consentement à payer de l'individu est nul et 0 sinon, et $\gamma=(\beta, \sigma)$. $\Phi($.$) est la fonction de répartition de la$ loi normale et $\phi($.$) sa densité. Trois modèles sont estimés par maximum de vrai-$ semblance sur la base des réponses aux questions ouvertes : CAP sans information, CAP avec information et CAP spécifique à la mortalité.

Certains caractères spécifiques à l'enquête ont nécessité des adaptations particulières. Tout d'abord, la méthodologie adoptée est fondée sur deux types d'enquête : téléphonique et simultané dans l'hémicycle de l'hôtel de région. Il semble donc logique de supposer que le mode d'administration des questionnaires ait une influence sur les consentements à payer révélés (Mitchell et Carson [1989]). En conséquence, il est nécessaire de prendre en compte l'influence potentielle du mode d'administration, ce qui est fait par l'introduction de termes constants et d'écarts types différents selon que l'individu était interrogé par téléphone ou lors de sessions en simultané. De plus, dans le cas de l'enquête téléphonique, les questionnaires devaient être suffisamment courts pour être renseignés en une vingtaine de minutes. Pour ce faire, il a donc fallu adopter un 
mécanisme de révélation combinant questions ouvertes et questions fermées, cette dernière étant susceptible de générer un effet d'ancrage sur l'offre de départ. Un paramètre permettant de capturer un biais d'ancrage potentiel a donc été introduit dans la spécification pour les individus interrogés par téléphone.

\section{Estimation des CAP pour l'ensemble des effets associés à la pollution atmosphérique}

Parmi les 1273 personnes de l'échantillon initial, 978 réponses sont utilisables du fait de données manquantes pour certaines variables déterminantes comme le revenu d'une part, et du fait de certains refus de réponse aux questions de valorisation, d'autre part. Sur l'ensemble de l'enquête, seules 101 personnes (soit 7,9\%) ont refusé de répondre. Ce taux de non-réponses est relativement peu élevé par rapport à d'autres études sur la pollution de l'air (Rozan [1996] et Halvorsen [1996]) qui avaient utilisé des scénarios fondés sur des décisions portant sur des politiques publiques. Ce premier résultat confirme la pertinence d'un scénario exprimé en termes de décision privée pour garantir un taux de réponse élevé.

Les deux premières colonnes du tableau en annexe $\mathrm{C}$ présentent les résultats obtenus pour deux consentements à payer déclarés : le consentement à payer sans information et le consentement à payer après apport d'information. Les deux modèles sont globalement très significatifs, les tests de nullité jointe donnant des valeurs critiques des probabilités inférieures à $10^{-6}$. On constate que ce sont quasiment les mêmes variables explicatives qui s'avèrent significatives. La variable âge, intégrée sous une forme polynomiale (la variable ellemême et son carré), s'est avérée statistiquement la plus pertinente et agit positivement sur le CAP jusqu'à 65 ans dans les deux modèles (avec un effet maximal à l'âge de 32 ans). Les niveaux de diplômes des répondants ont également un effet significatif sur les consentements à payer : un effet croissant avec le niveau de diplôme, l'individu de référence ayant un niveau de diplôme inférieur au baccalauréat. L'activité des personnes interrogées influence également le CAP, la référence étant constituée par les individus étudiants ou en formation.

Parmi l'ensemble des variables spécifiques à la pollution de l'air', seules deux s'avèrent significatives : percevoir la pollution de l'air à Marseille comme meilleure que dans les autres villes de France et avoir une bonne connaissance de la pollution de l'air. La première de ces variables a un effet négatif. Ce résultat n'est pas étonnant puisque les personnes qui pensent que le niveau de pollution 1'air :

1. Cinq questions permettaient de recueillir des informations sur la perception de la pollution de

1. Comment jugez-vous la qualité globale de l'air dans les Bouches-du-Rhône ?

2. Comparée à d'autres grandes villes françaises, pensez-vous que la qualité de l'air à Marseille est : Supérieure/Légèrement supérieure/Équivalente/Moins bonne/Très inférieure/Ne sait pas.

3. Comparée à Marseille, pensez-vous que la qualité de l'air dans votre lieu de résidence (quartier) est : Supérieure/Légèrement supérieure/Équivalente/Moins bonne/Très inférieure/Ne sait pas.

4. Est-ce qu'il vous est déjà arrivé de ressentir personnellement la pollution de l'air ? (mauvaises odeurs, aspects visuels, irritation des yeux, difficultés à respirer ou autre).

5. Vous-même ou un de vos proches (famille, ami) a-t-il eu des problèmes de santé liés à la pollution de l'air?

L'annexe B présente une analyse quantitative détaillée des écarts entre la pollution perçue et la pollution objective. 
à Marseille est meilleur que dans les autres villes de France sont moins enclines à payer pour un niveau de pollution relativement bon. Cette variable suggère ainsi que les perceptions de la pollution de l'air des individus vont influer sur les montants révélés et ce, malgré un apport d'information objective sur les conséquences de la pollution en termes de morbidité et de mortalité puisque cette variable reste significative dans le second modèle (CAP avec information en annexe C). La seconde variable agit, elle, positivement : un individu déclarant avoir une bonne connaissance de la pollution de l'air a un consentement à payer supérieur. On peut penser que ces individus, puisqu'ils se tiennent informés, se sentent plus préoccupés par les problèmes associés à la pollution de l'air.

Le fait d'appartenir à une association de protection de l'environnement affecte négativement le consentement à payer révélé. Ce résultat peut paraître étonnant puisque les personnes impliquées dans un mouvement écologique devraient avoir un consentement plus élevé. On peut toutefois supposer qu'elles considèrent qu'il revient aux responsables de la pollution de payer, ce qui peut les conduire à déclarer un CAP inférieur. Être non-fumeur joue un rôle positif sur les CAP. Cet effet peut être attribué à un simple effet revenu lié à la part des dépenses de tabac dans le budget du ménage. Toutefois, les conséquences en termes de santé de la pollution de l'air (morbidité et mortalité) étant essentiellement d'ordre respiratoire et cardiovasculaire, on peut supposer que les individus non fumeurs sont plus sensibles à une amélioration de la qualité de l'air, et donc de leur santé, que les répondants fumeurs, moins susceptibles de bénéficier d'une telle amélioration.

Le paramètre $\alpha$, qui mesure l'élasticité du CAP par rapport au revenu, est très significatif et vaut respectivement 0.174 et 0.186 pour le CAP sans information et avec information. Ce résultat est conforme aux résultats de McFadden et Léonard [1993] qui obtiennent une valeur de 0.27 en analysant dans un même modèle économétrique plusieurs modes d'élicitation. Le coefficient étant très significativement différent de zéro, le CAP augmente donc avec le niveau de revenu, gage de la validité interne des résultats puisque conforme aux prédictions de la théorie économique. Une valeur inférieure à 1 implique que les ménages riches consentiront cependant à payer une part moins importante de leur revenu que les ménages moins fortunés. Le paramètre relatif au biais d'ancrage est également significatif pour les deux CAP, témoignant d'un effet de l'offre de départ sur les CAP. Cependant, cet effet reste relativement limité et décroît pour le CAP avec information : ainsi, dans une procédure itérative, l'effet de la première offre semble s'atténuer au cours des différentes questions de valorisation.

Sur la base de ces estimations et des caractéristiques individuelles de l'échantillon, il est possible de calculer les CAP moyens avec et sans apport d'information. Ils s'élèvent respectivement, pour une diminution de $50 \%$ de la pollution de l'air tous effets confondus, à $427 \mathrm{~F}$, soit $\mathbf{6 5}$ euros (écart type : $224 \mathrm{~F}$ ) et à $457 \mathrm{~F}$, soit 69,7 euros (écart type : $218 \mathrm{~F}$ ) par mois et par ménage. L'apport d'information se traduit ainsi par une augmentation de 4,7 euros en moyenne, soit $7 \%$. Les données agrégées ne peuvent cependant qu'imparfaitement représenter l'impact de l'information au niveau individuel. L'analyse d'un tel effet nécessiterait l'utilisation d'un modèle économétrique spécifique (de type panel ou équations simultanées) ${ }^{1}$.

1. Voir Luchini et al. [2003]. 
Ces consentements à payer moyens concernent la valorisation de l'ensemble des effets de la pollution atmosphérique. Un des objectifs de cette recherche porte sur le consentement à payer spécifique à la mortalité engendrée par la pollution. Dans cette perspective, un sous-échantillon de la population enquêtée a été interrogé et leurs réponses, replacées dans un cadre théorique adapté, permettent d'établir une valeur d'évitement d'un décès (VED) contextuelle. Ceci est l'objet de la section suivante.

\section{Estimation d'une valeur d'évitement d'un décès contextuelle}

Afin d'estimer une VED contextuelle utilisable dans le calcul économique, il convient de replacer dans un cadre formel le montant monétaire déclaré auquel est associée la variation de probabilité de décès. À cet effet, nous présentons un modèle d'optimisation d'utilité avec durée de vie aléatoire dont nous définissons les conditions d'application avant de l'utiliser sur les CAP portant uniquement sur la mortalité.

\section{Cadre théorique et conditions d'application}

Dans le cadre théorique présenté en annexe D, la VED d'un individu d'âge $j$, $\mathrm{VED}_{j}$, s'écrit comme

$$
\operatorname{VED}_{j}=\frac{1}{\lambda^{*} e^{-r j}} \int_{j}^{\mathrm{T}} \mathrm{S}_{t, j}\left[u\left(c_{t}^{*}\right) e^{-\delta(t-j)}+\lambda^{*} e^{-r(t-j)}\left(y_{t}-c_{t}^{*}\right)\right] \mathrm{d} t
$$

où $\lambda$ est l'utilité marginale de la monnaie, $r$ le taux d'intérêt d'un actif sans risque, T représente l'âge maximal que peut atteindre un être humain, $\mathrm{S}_{t, j}$ la probabilité de survie à l'âge $t$ conditionnellement au fait d'être vivant à l'âge $j$, $u\left(c_{t}\right)$ est l'utilité de la consommation à l'âge $t, \delta$ représente le taux individuel de préférence pour le présent, $y_{t}$ le revenu à l'âge $t$ et l'exposant ${ }^{*}$ caractérise les valeurs résultant du processus d'optimisation. Nous montrons, en annexe D, que cette valeur d'évitement d'un décès peut également s'écrire comme :

$$
\mathrm{VED}_{j}=(\mathrm{D} d \mu)^{-1} \mathrm{CAP}_{j}
$$

où $d \mu$ est la variation de la probabilité de décès, $\mathrm{D}$ la durée de cette variation et $\mathrm{CAP}_{j}$ le consentement à payer à l'âge $j$. Dans la mesure où l'on souhaite se départir d'une forme particulière de la fonction d'utilité $u($.$) et d'hypothèses sur$ $c_{t}^{*}, \mathrm{D}, r$ et $\delta$, pour calculer la VED d'un individu d'âge $j$, nous retenons la formulation (8) : seuls les montants du $\mathrm{CAP}_{j}$ et la variation de la probabilité de décès $d \mu$ sont alors nécessaires ${ }^{1}$.

Avant d'appliquer ce modèle aux données d'enquête, il faut alors préciser : la durée de la réduction de la mortalité ; la détermination de la variation de risque

1. Il est également possible d'exprimer cette VED comme un flux d'années de vie perdues actualisées :

$$
\operatorname{VED}_{j}=\int_{j}^{\mathrm{T}} e^{-\delta(t-j)} \mathrm{S}_{t, j} \operatorname{VOLY}_{t} \mathrm{~d} t
$$

où $\operatorname{VOLY}_{t}$, qui représente la valeur d'une année de vie perdue à l'âge $t$, peut éventuellement dépendre de l'âge. 
de décès pour la population concernée $d \mu$; le montant monétaire individuel $\mathrm{CAP}_{j}$.

Durée de la réduction. Dans le cadre théorique général, on considère que la personne est prête à payer en une seule fois le montant monétaire annuel qui compense la variation d'utilité espérée relative à la variation de la probabilité de survie $d \mathrm{~S}_{t, j}$ durant la période $\mathrm{D}$. Le scénario utilisé dans les enquêtes proposait une somme mensuelle, qui est donc multipliée par 12 pour obtenir un CAP annuel. Ce paiement se répétera tant que l'individu bénéficiera de cette variation de probabilité de décès $d \mu$ pendant la période $\mathrm{D}$. Nous choisissons de raisonner avec $\mathrm{D}=1$ et devons donc calculer le $d \mu$ correspondant.

Expression de la variation de la probabilité de décès $d \mu$. La variation de risque de mortalité est exprimée dans le scénario sur une période de temps supérieure à l'année, afin de ne pas raisonner sur de trop faibles probabilités (pour le type de risque considéré, de l'ordre de $10^{-4}$ par an). Elle a donc été exprimée comme « deux personnes de la ville polluée sur cent prises au hasard dans la rue (autrement dit, d'âge médian) décéderont du fait d'une exposition à la pollution atmosphérique avant d'avoir 80 ans ». On propose à l'individu de réduire ce risque en déménageant dans la ville moins polluée. Chaque individu est confronté, sur la base d'un tirage aléatoire, à un niveau de réduction parmi les trois niveaux suivants : $25 \%, 50 \%$ ou $100 \%$. On détermine alors la variation de la probabilité de décès $d \mu$ qui résulte de la variation du risque du scénario proposé, à partir d'une forme paramétrique de la fonction de survie conditionnelle (voir calculs en annexe E). Pour une réduction de moitié du nombre de jours pollués, $d \mu^{8}=0,0004328$, pour une réduction totale, $d \mu^{16}=0,0008378$ et pour une réduction d'un quart, $d \mu^{4}=0,00022$.

Détermination d'une VED au niveau individuel. Le scénario demande la somme monétaire nécessaire pour que le répondant déménage avec les personnes habitant avec lui, et concerne donc une variation du risque de mortalité pour l'ensemble des membres du ménage. L'estimation de la VED nécessite d'obtenir un CAP individuel. Les études recourant aux méthodes hédonistiques pour évaluer une VED utilisent en règle générale le nombre de personnes composant le ménage sans pondération particulière, alors que certains travaux empiriques en macroéconomie pondèrent parfois différemment les mineurs en fonction de leur âge. Nous retenons, pour notre part, une pondération unitaire pour chaque membre du ménage.

\section{Estimation d'une VED individuelle}

Un sous-échantillon de 923 individus a été interrogé sur un CAP spécifique à la seule réduction du risque de mortalité, conduisant à un échantillon exploitable de 759 individus. Le même modèle économétrique que précédemment est estimé et ses résultats sont présentés dans la troisième colonne du tableau 1 en annexe $\mathrm{C}$.

En premier lieu, le test de nullité jointe de tous les paramètres conduit à une valeur critique de $10^{-5}$. Le modèle global est donc très significatif. La variable âge est intégrée comme précédemment sous une forme polynomiale. Elle agit de façon significative, et possède un effet positif sur le CAP jusqu'à 75 ans (avec un maximum à l'âge de 38 ans). L'effet du niveau de diplôme des répondants sur le CAP est également croissant. Concernant l'activité des personnes interrogées, seuls les inactifs (dont 65,4\% de retraités) influencent positivement et significa- 
tivement le CAP. Parmi les variables spécifiques à la pollution de l'air, seule la perception de la qualité de l'air à Marseille reste significative. Elle affecte encore, comme précédemment, le CAP à la baisse. Être non-fumeur joue toujours un rôle positif sur les CAP, alors que l'effet de l'ensemble des autres variables socio-économiques apparaît comme non significatif. Le paramètre d'élasticité $\alpha$, très significatif, est égal à 0.217 : la part du revenu consacrée à la réduction du risque de mortalité s'accroît donc plus sensiblement avec le niveau de revenu que pour une réduction de l'ensemble des effets sanitaires et non sanitaires. Le paramètre relatif au biais d'ancrage reste significatif, mais décroît sensiblement $(0.12$ contre 0.22 pour le CAP sans information) et perd de sa significativité $(7,2 \%$ au lieu de 1,1\%). Ce dernier résultat confirme l'affaiblissement de l'effet d'ancrage sur l'offre de départ dans la procédure itérative de révélation des CAP.

Le CAP mensuel moyen pour diminuer les effets mortels associés à l'exposition à la pollution atmosphérique s'élève à $405 \mathrm{~F}$, soit 61,7 euros (écart type 210), soit $90 \%$ de la valeur obtenue pour réduire l'ensemble des effets. Ceci confirme la place importante qu'occupe, au niveau individuel, la mortalité parmi les bénéfices à attendre d'une politique de réduction. La valeur d'évitement d'un décès est calculée à partir des CAP individuels estimés (annualisés et divisés par le nombre de personnes composant le foyer) et de la variation de risque correspondante $d \mu$. Sur la base de la distribution de ces VED individuelles, on peut évaluer la VED moyenne à 5,25 millions (M) de francs 2001, soit $0,8 \mathrm{M}$ d'euros (écart type 4.63) et la médiane à 4,1 M de francs, soit 0,625 $\mathrm{M}$ d'euros. On constate que $90 \%$ des VED estimées se trouve entre 0,13 et $21 \mathrm{M}$ d'euros.

Bien qu'aucune évaluation contingente contextuelle à la pollution atmosphérique n'existe, il est toutefois intéressant de comparer la valeur de $0,8 \mathrm{M}$ d'euros à d'autres valeurs de la littérature. En utilisant la MPP, Masson [1996] propose une valeur de 0,18 M d'euros (en 1995) pour la France, et Ecoplan [1996] 0,3 M d'euros (en 1993) pour la Suisse, toutes les deux étant indépendantes de l'âge et du sexe. En travaillant sur des risques professionnels (méthode de valorisation ex post), Moore et Viscusi [1988] obtiennent des valeurs associées à un décès comprises entre 2,1 et 6,7 M d'euros [1996], et Lanoie et al. [1995], autour de 10 millions.

Par correction ex post d'une valeur non contextuelle obtenue par MEC, Ostro et Chestnut [1998] tiennent compte de l'âge moyen de décès en appliquant un coefficient de 0.8 , et proposent 3,1 M d'euros 2000 comme valeur centrale. Pearce et Crowards [1996] appliquent un coefficient de 0.7 et préconisent 2,1 M d'euros, et Sommer et al. [1999] appliquent un ajustement pour l'âge moyen de décès de 0.61 , et utilise $0,9 \mathrm{M}$ d'euros dans une étude européenne. Certains auteurs vont plus loin encore, et appliquent une correction pour l'âge et la qualité de vie au moment du décès : Leksell [2000] applique ainsi un facteur de correction de 0.7 pour l'âge et la qualité de vie à une VED issue d'une MEC sur les accidents de la route, et retient la valeur de 1,45 M d'euros. Krupnick et al. [2002] interrogent des individus de 40 ans et plus, population la plus touchée par les affections associées à la pollution atmosphérique, et obtiennent des VED variant entre 0,71 et 2,4 M d'euros. L'impact de l'âge sur la VED répondant à une forme quadratique, ils proposent une VED de 0,5 M d'euros pour les âges supérieurs à 70 ans.

Ainsi, la valeur contextuelle de $0,8 \mathrm{M}$ d'euros se situe plutôt dans la borne inférieure de la fourchette et, si elle venait à être confirmée par les résultats d'autres études contextuelles, plaiderait pour une révision de la VED utilisée dans les procédures d'évaluation publique relatives à la pollution de l'air. 


\section{CONCLUSION}

Cet article s'attache à montrer l'influence, à l'échelon territorial, des représentations du risque et des caractéristiques individuelles sur la valorisation des composantes non marchandes associées à une détérioration du bien-être individuel liée à la pollution atmosphérique. Il repose sur une analyse d'entretiens, sur une enquête novatrice d'évaluation contingente simultanée et sur une enquête plus traditionnelle par téléphone.

Une attention particulière a été portée à la prise en compte des biais usuels, que ce soit dans la construction du questionnaire ou dans le traitement économétrique. Les résultats montrent que l'acceptabilité du thème, du questionnaire et des scénarios proposés a été excellente, comme l'indique le faible taux de refus de réponses (moins de $8 \%$ contre 20 à $25 \%$ dans les enquêtes usuelles). Un cadre économétrique adapté traite de façon différenciée les valeurs nulles par un modèle avec censure, prend en compte la possible influence des valeurs de départ sur les réponses, et utilise une forme fonctionnelle de type Box-Cox susceptible de prendre en compte divers types de non-linéarités. L'analyse économétrique du CAP portant sur l'ensemble des effets négatifs associés à la pollution atmosphérique apporte des informations précieuses. L'influence de certaines variables sur les CAP est apparue de manière récurrente : l'effet positif du revenu sur le CAP, l'effet quadratique de l'âge sur la valeur du CAP, les effets du niveau de diplôme et de l'activité professionnelle ainsi que celui d'être non fumeur. Déclarer avoir une bonne connaissance de la pollution de l'air et avoir changé ses habitudes à cause de la pollution jouent également favorablement sur le montant du CAP. Le CAP mensuel moyen par ménage pour une réduction de moitié du nombre de jours pollués est estimé à 65 euros. La question de l'information prend une place d'autant plus importante dans la méthode d'évaluation contingente que, à la différence des marchés sur lesquels les prix d'équilibre véhiculent l'ensemble de l'information sur la rareté relative des biens, le scénario hypothétique contient ici toute l'information. Dans cette étude, l'apport d'une information sur les effets de la pollution atmosphérique exprimés se traduit par un accroissement de $7 \%$ des valeurs monétaires.

L'application de ce type de méthode n'échappe toutefois pas à un certain nombre de critiques. En premier lieu, comme tout scénario hypothétique, le cadre choisi dans cette étude soulève certaines limites inhérentes à la méthode d'évaluation contingente. En effet, en choisissant une ville de référence commune à l'ensemble de la population (Marseille), le scénario peut générer des biais de perception au sein de la population. Les individus qui perçoivent imparfaitement la pollution de l'air dans leur lieu de résidence (annexe B) peuvent éventuellement avoir une perception erronée de la pollution de l'air à Marseille. Et plus généralement, les individus peuvent avoir une représentation des deux lieux proposés qui ne se restreigne pas à leurs seules différences de niveau de pollution et de coût de la vie. Ces biais de perception ne sont cependant pas spécifiques à la pollution de l'air mais peuvent apparaître dans toute évaluation contingente. En second lieu, bien que nous ayons eu le souci de présenter les conséquences de la pollution, tant en termes de morbidité que de mortalité, avec des échelles que les individus peuvent plus facilement se représenter, il n'en demeure pas moins que les risques sous-jacents restent de «faibles risques » pouvant conduire à des difficultés d'appréciation. Enfin, malgré ses vertus 
démocratiques, la méthode d'évaluation contingente ne garantit pas que les individus se comportent strictement tels des consommateurs face à la transaction hypothétique proposée et ce, en dépit des attentions apportées à la définition du scénario. La variable appartenir à une association environnementale montre en effet que tous les biais n'ont peut-être pas été éliminés ${ }^{1}$.

Un des objectifs majeurs de cette recherche concernait l'évaluation d'une valeur d'évitement d'un décès (VED) contextuelle, c'est-à-dire établie sur la base des révélations de préférences individuelles spécifiques à une variation de risque de mortalité associée à la pollution atmosphérique. Le cadre théorique retenu, qui nécessite bien évidemment un certain nombre d'hypothèses, permet néanmoins de traduire les comportements individuels sous-jacents. La valeur obtenue par le modèle explicatif est proche de 0,8 million d'euros. Cette première VED dans un cadre contextuel intégré peut être considérée comme un ordre de grandeur raisonnable pour valoriser un décès attribué à une exposition prolongée à la pollution atmosphérique.

\section{RÉFÉRENCES BIBLIOGRAPHIQUES}

Arrow K., Solow R., Portney P. R., LeAmer E. E., RADNER R. et SCHUMAN H. [1993], « Report of the NOAA panel on contingent valuation », Technical Report, 58, p. 1601-1614.

BLOMQUIST G. C. et WHITEHEAD J. C. [1991], « Measuring contingent Values for Wetlands: Effects of information about related environmental goods », Water Resource Research, 2.

BLOMQVIST A. [2002], « Defining the value of a statistical life: a comment », Journal of Health Economics, 21, p. 169-175.

BOYLE K. [1994], "An investigation of part-whole biases in contingent-valuation studies », Journal of Environmental Economics and Management, 27, p. 64-83.

Chanel O., Faugère E., Geniaux G., Kast R., Luchini S., Protopopescu C. et SCAPECCHI P. [2001], « Approche socio-anthropologique et économique de la pollution atmosphérique: une application au département des Bouches-du-Rhône », Rapport pour le ministère de l'Environnement et de l'Aménagement du territoire, Rapport Primequal 36/98, décembre.

Chanel O., Geniaux G., Rychen F., Deniau C. et Ghattas B., [1996], «Évaluation monétaire des effets à court terme de la pollution atmosphérique sur la santé, application à l'Ile-de-France », Marseille, GREQAM, Rapport de recherche, Étude 95270 pour le ministère de l'Environnement.

COMMON M., REID I. et BLAMEY R. [1997], « Do existence value for cost benefit analysis exist? », Environmental and Resource Economics, 9, p. 225-228.

DIAMOND P.A. et HAUSMAN J.A. [1993], « On contingent valuation measurement of nonuse values », dans HAUSMAN J.A. (ed.), Contingent Valuation: A Critical Assessment, North-Holland.

ECOPLAN [1996], «Monétarisation des coûts externes de la santé imputables aux transports », Rapport - mandat SET 272, Berne.

FAUGÈRE E. [2002], « Percevoir ou mesurer ? Approche anthropologique de la qualité de l'air », EUROPAEA, Journal des européanistes, VIII-1.

Filliger P., PuybonNieUX-TÉXIER V. et SCHNEIDER J. [1999], « Health costs due to road traffic-related air pollution. An impact assessment project of Austria, France and Switzerland - PM10 population exposure », Rapport technique OMS n ${ }^{\circ}$ TEH05.

1. Voir Luchini [2003] pour une discussion. 
Green D., Jacowitz K.E., KAhNeman D. et MCFAdDEn D. [1998], «Referendum contingent valuation, anchoring, and willingness to pay for public goods », Resource and Energy Economics, 20, p. 85-116.

HALVORSEN B. [1996], « Ordering effects in contingent valuation surveys: willingness to pay to reduce health damage from air pollution », Environmental and Resources Economics, 8, p. 485-499.

HANLEY N. D. et MunRo A. [1992], « The effects of information in contingent markets for environmental goods: A survey and some new evidence », Papers 848, Queen's at Kingston - Institute for Economic Research.

Hausman J. A. (ed.) [1993], Contingent Valuation: A Critical Assessment, Contribution to Economic Analysis, vol. 220 of Contribution to Economic Analysis, Amsterdam, North-Holland.

HERRIGES J. et SHOGREN J. [1996], « Starting point bias in dichotomous choice valuation with follow-up questioning », Journal of Environmental Economics and Management, 30, p. 112-131.

HOLLAND M. et KING K. [1998], « Economic evaluation of air quality targets for tropospheric ozone », Final report + 3 reports, Rapport pour l'UE, 4 vol., novembre, contract: B4-3040/97/000654/MAR/B1.

HollAND M et KING K. [1999], «Economic evaluation of a directive on national emission ceilings for certain atmosphere pollutants, Part B: Benefit Analysis », Rapport DGXII, novembre.

INSEE [1997], «La situation démographique », Démographie-Société, p. 65-66.

JOHANSSON P.O. [2001], «Is there a meaningfu definition of the value of a statistical life », Journal of Health Economics, 20, p. 131-139.

JONES-LEE M. et LOOMES G. [1999], The monetary valuation of safety and its role in the appraisal of proposed air abatement programmes, miméo, CASPAR, University of Newtcastle upon Tyne.

KEMP M.A. et MAXWELl C. [1993], Budget Context for CV Estimates, vol. Contingent Valuation: a Critical Assessment, p. 217-265, New York, North-Holland, Hausman.

Künzli N., Kaiser R., Medina S., Studnicka M., Chanel O., Herry M., HoraK F., PuybonNieuX-TEXIER V., QUENEl P., SCHNEIDER J., SEETHALER R., VERGNAUd J.-C. et SOMMER H. [2000], « Public-health impact of outdoor and traffic-related air pollution: a european assessment », Lancet, 356, p. 795-801.

KoTLIKOFF L. J. et SPIVAK A. [1981], « The family as an incomplete annuities market », Journal of Political Economy, 89, p. 372-391.

Krupnick A.J., Alberini A., Cropper M., Simon N., O’Brien B., Goeree R. et HEINTZELMAN M. [2002], «Age, health and the WTP for mortality risk reductions: a CV survey of Ontario residents », The Journal of Risk and Uncertainly, 24 (2), p. 161186.

LANDEFELD J.S. et SESKIN E.P. [1982], « The economic value of life: linking theory and practice », American Journal of Public Health, 72, p. 555-666.

LANOIE P., PEDRO C. et LATOUR R. [1995], « The value of a statistical life: a comparison of two approaches », Journal of Risk and Uncertainty, 10, p. 235-257.

LEKSELL I. [2000], « Health costs of particle emissions-economic valuation of increaed mortality due to exhaust emissions of fine particles », Chalmer University of Technology, Göteborg University, Sweden, Department of physical resource theory - Goteborg University.

LEKSELL I. et RABL A. [2001], « Air pollution and mortality: Quantification and valuation of years of life lost », Risk analysis, 21, p. 843-857.

LUCHINI S. [2003], « De la singularité de la méthode d'évaluation contingente », Économie et Statistique, 357-358, p. 141-152.

LuChini S., ProtiÈre C., DonaldSON C. et MOATTI J-P. [2003], « Contingent valuation of multiple health programs: major improvements or major biaises? », DT GREQAM, Marseille.

MASSON S. [1996], « Faisabilité d'une évaluation des coûts de la pollution atmosphérique sur la santé en Ile-de-France », Mémoire de DEA, juin, BETA, Strasbourg. 
MCFADDEN D. et LEONARD G. [1993], Issues in the contingent valuation of environmental goods: Methodologies for data collection and analysis, vol. Contingent Valuation: a Critical Assessment, p. 165-215, New York, North-Holland, Hausman.

Mitchell R.C. et CARSON R.T. [1989], Using Surveys to Value Public Goods: The Contingent Valuation Method, Washington DC, Resources for the Future.

MoORE M.J. et VISCUSI W.K. [1988], « Doubling the estimated value of life: results using new occupational fatality data, Journal of Policy Analysis and Management, 7, p. 476-490.

OSTRO B. et CHESNUT L. [1998], « Assessing the health benefits of reducing particulate matter air pollution in the United States », Environmental Research, 76, p. 94-106.

PALMQuist R. [1991], « Hedonic methods », dans BRADEN J. et Kolstad C. (ed.), Measuring the demand for environmental improvement, Amsterdam, Elsevier.

PEARCE D. et CROWARDS T. [1996], « Particulate matter and human health in the United Kingdom », Energy Policy, 24, p. 609-619.

RoZAN A. [1996], «Évaluation contingente des bénéfices de santé d'une amélioration de la qualité de l'air: l'exemple de la région strasbourgeoise », Thèse de doctorat, Université Louis-Pasteur, Strasbourg.

SAGOFF M. [1998], "Aggregation and deliberation in valuing environmental public goods: a look beyond contingent pricing », Ecological Economics, 24, p. 213-230.

SHEPARD D.S. et ZECKHAUSER R.J. [1984], « Survival versus consumption », Management Science, 30, p. 93-142.

Sommer H., Seethaler R., Chanel O., Herry M., Masson S. et Vergnaud J-C. [1999], « Health costs due to road taffic-related air pollution », Technical Report TEH07 on Economy prepared for the WHO Ministerial Conference on environment and health, Londres, juin.

TVERSKY A. et KAHNEMAN D. [1981], « The framing of decisions and the psychology of choice », Science, 211, p. 453-458.

UK, Department of Health [1999], «Economic appraisal of the health effects of air pollution », The Stationery Office. 


\section{ANNEXES}

\section{A. SCÉNARIO HYPOTHÉTIQUE ET QUESTIONS DE RÉVÉLATION DES CAP}

Le scénario et les questions proposées aux individus sont reproduits ci-dessous. Les phrases en italique sont des commentaires à destination du lecteur, non lus aux répondants.

Nous allons vous proposer un scénario dans lequel vous êtes l'acteur principal. Vous allez devoir prendre la décision qui vous semble la meilleure pour vous et les membres de votre famille qui habitent le même logement que vous.

Supposons que vous et les membres de votre famille qui habitent avec vous soyez obligés de déménager. Vous avez le choix entre deux lieux de résidence, équivalents à tous les points de vue (nombre d'habitants, conditions de travail, écoles, climat, équipements publics, vie culturelle, transports, logement, ambiance, etc.) Une chose seulement est différente entre ces deux lieux : le niveau de pollution atmosphérique. Le premier lieu (que l'on appelle POL) subit le même nombre de jours de pollution que la ville de Marseille, et le second lieu (que l'on appelle PEUPOL) subit moitié moins de jours de pollution.

Le problème est que la vie est plus chère à PEUPOL (la ville la moins polluée) : le logement, les taxes locales, les transports publics, etc., vous coûteront plus chers. Cela veut dire que si vous choisissez de déménager vers PEUPOL, vous devrez payer une somme supplémentaire pour avoir le même niveau de vie qu'à POL.

\section{Scénario sans information}

Ce que l'on désire connaître, c'est la somme mensuelle que vous accepteriez de payer au maximum actuellement pour que vous-même et les membres de votre famille habitant avec vous déménagiez vers PEUPOL (la ville la moins polluée) plutôt que vers POL (la ville polluée comme Marseille). N'oubliez pas que cette somme supplémentaire sera prélevée sur le budget de votre ménage, et qu'il vous restera donc moins d'argent à la fin du mois.

QUESTION 1. Pour que vous et les membres de votre famille qui habitent avec vous déménagiez vers PEUPOL (la ville la moins polluée) plutôt que vers POL (la ville polluée comme Marseille), accepteriez-vous de payer au moins :

Les enquêtés de l'hémicycle du conseil régional ont répondu par boîtier de vote électronique Oui, Non ou Ne sait pas, à chacune des valeurs suivantes, proposées oralement et apparaissant sur un écran individuel :

$10,50,100,200,400,700,1000,1500$ et plus nouveaux francs par mois, soit par an, 120, 600, 1 200, 2 400, 4 800, 8 400, 12 000, 18000 et plus?

Les enquêtés par voie téléphonique ont été soumis de façon aléatoire à une des trois double enchère suivante :

$\mathrm{X}$ nouveaux francs par mois ? Si oui : Seriez-vous prêt à payer Y nouveaux francs par mois ? Si non: Seriez-vous prêt à payer $\mathrm{Z}$ nouveaux francs par mois ?

avec $(\mathrm{X}, \mathrm{Y}, \mathrm{Z})$ respectivement $(300,800,50),(400,1000,100)$ et $(500,1200,200)$.

QUESTION 2. Quelle est en fait la somme approximative que vous seriez prêt à payer par mois pour habiter dans la ville a moins polluée ? ......... Francs par mois. 


\section{Scénario avec apport d'information}

En fait, très peu de personnes connaissent bien les effets de la pollution atmosphérique. Ces effets se décomposent en trois catégories : les effets purement polluants, les effets irritants et les effets mortels.

Les effets purement polluants encrassent les bâtiments, qui doivent être nettoyés plus souvent, sentent mauvais et se traduisent par un nuage de poussières de couleur marron.

Les effets irritants provoquent des maladies supplémentaires : yeux irrités, maux de tête et de gorge, épisodes de toux, symptômes qui ressemblent à la grippe et même des admissions à l'hôpital pour des problèmes respiratoires et cardiaques.

Les effets mortels raccourcissent la durée de vie. En effet, l'exposition pendant plusieurs années à un niveau de pollution élevé conduit à une dégradation de l'état de santé, et donc à des décès prématurés. Pour donner un ordre de grandeur, sur 100 personnes prises au hasard et habitant PEUPOL, UNE personne décédera avant d'avoir 80 ans à cause de complications de son état de santé provoquées par la pollution. Elle perdra environ dix années de vie. Si ces 100 personnes habitaient POL, DEUX personnes décéderaient. Donc, une personne sur 100 peut vivre environ dix ans de plus en habitant à PEUPOL plutôt qu'à POL.

Maintenant que vous connaissez mieux les effets de la pollution, nous vous redemandons :

QUESTION 3. Quelle est la somme mensuelle maximale que vous accepteriez de payer actuellement pour que vous et les membres de votre famille qui habitent avec vous déménagiez vers PEUPOL (la ville la moins polluée) plutôt que vers POL la ville polluée comme Marseille) : ......... Francs par mois.

\section{Scénario avec suppression des effets mortels uniquement}

Supposons, pour finir, que seuls les effets mortels de la pollution atmosphérique soient diminués à PEUPOL. Cela veut dire qu'à PEUPOL, il y aura les mêmes effets irritants (causant des maladies supplémentaires) et les mêmes effets purement polluants qu'à POL. Par contre, une personne sur 100 pourra vivre environ dix ans de plus.

QUESTION 4. Sachant cela, quelle est la somme mensuelle maximale que vous accepteriez de payer actuellement pour que vous et les membres de votre famille qui habitent avec vous déménagiez vers PEUPOL (la ville a moins polluée) plutôt que vers POL (la ville polluée comme Marseille) : Francs par mois.

\section{B. MESURE DE LA POLLUTION ET PERCEPTIONS INDIVIDUELLES}

La zone géographique retenue est l'ensemble du département des Bouches-du-Rhône, qui constitue la deuxième concentration française d'installations industrielles présentant des risques majeurs pour le milieu naturel (dites «SEVESO »), principalement en raison du complexe industriel Fos-Berre. Une activité industrielle concentrée, un fort taux d'urbanisation, un réseau routier dense et des conditions climatiques locales spécifiques concourent à une qualité de l'air médiocre dans le département. L'analyse anthropologique démontre largement l'influence des perceptions sur les modes de représentations (Faugère [2002]). Le mode de construction du scénario nécessite alors de mesurer l'écart entre la perception subjective qu'ont les individus de la pollution atmosphérique et le niveau de pollution mesuré par les capteurs. 
Figure 1. Qualité de l'air d'après les réseaux de mesure dans les communes du département

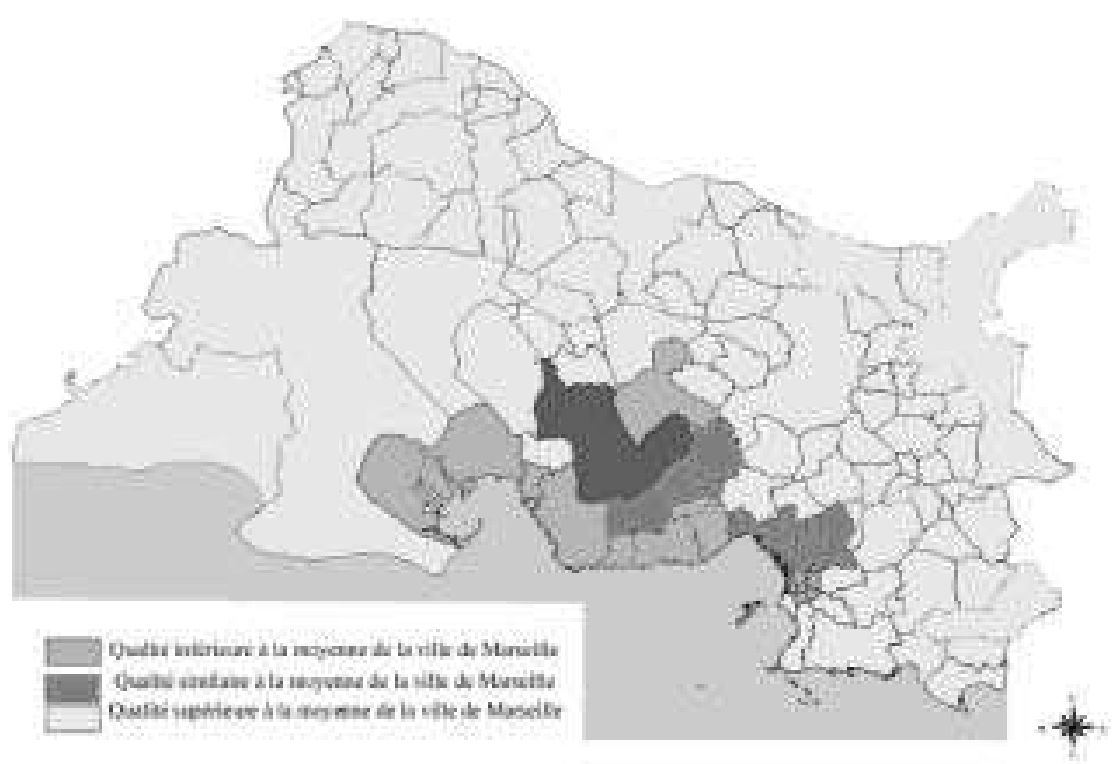

Pour comparer les mesures techniques avec les perceptions sensorielles, nous avons pris une référence commune à tous les individus enquêtés : la ville de Marseille. La mesure dite technique est fondée sur les réseaux agréés de surveillance de la qualité de l'air pendant l'année civile 2000 et concerne les trois indicateurs de pollution alors réglementés au niveau européen : le $\mathrm{SO}_{2}$, le $\mathrm{NO}_{2}$ et l'O $\mathrm{O}_{3}$. Un indice synthétise, pour chacune des 119 communes du département, la qualité de l'air relativement à la moyenne des 16 arrondissements de Marseille ${ }^{1}$. La figure 1 représente cet indice pour chacune des communes, classifié en trois niveaux : qualité inférieure, similaire ou supérieure à la moyenne (des 16 arrondissements) de Marseille. On constate que les communes accueillant les complexes industriels les plus importants (Fos-sur-Mer, Berre-l'Étang, Martigues) ainsi que le littoral entre Fos et Marseille qui se trouve sous le vent dominant des industries de Fos-sur-Mer, présentent une qualité de l'air inférieure à la moyenne de Marseille, essentiellement du fait de la pollution soufrée. Le centre de Marseille (du $1^{\text {er }}$ au $6^{\mathrm{e}}$ arrondt.) est également plus pollué que la ville dans son ensemble, essentiellement $\mathrm{du}$ fait de la pollution automobile (principal émetteur de $\mathrm{NO}_{2}$ ). Chateauneuf-les-Martigues, Vitrolles, Marignane et Rognac, en raison de leur proximité des sites industriels, et les arrondissements nord de Marseille $\left(\mathrm{du} 13^{\mathrm{e}}\right.$ au $\left.16^{\mathrm{e}}\right)$ présentent une qualité de l'air globalement comparable à la moyenne de Marseille. Enfin, le reste du département, moins peuplé et moins industriel, ainsi que les quartiers sud de Marseille, à l'habitat plus diffus, possèdent une qualité de l'air globalement supérieure à la moyenne de Marseille.

La seconde mesure est établie à partir de la perception par chaque individu de la qualité de l'air de son lieu de résidence comparée à celle de Marseille. La population enquêtée pense majoritairement (53\%) que la qualité de l'air de son lieu de résidence est supérieure à Marseille, $20 \%$ la considèrent comme équivalente et $24 \%$ comme inférieure.

1. La distribution des concentrations des trois indicateurs est divisée en cinq classes et l'indice synthétique est la somme de ces trois indicateurs discrétisés. 
Figure 2. Écart entre qualité de l'air mesurée et qualité de l'air perçue par les habitants des communes du département*

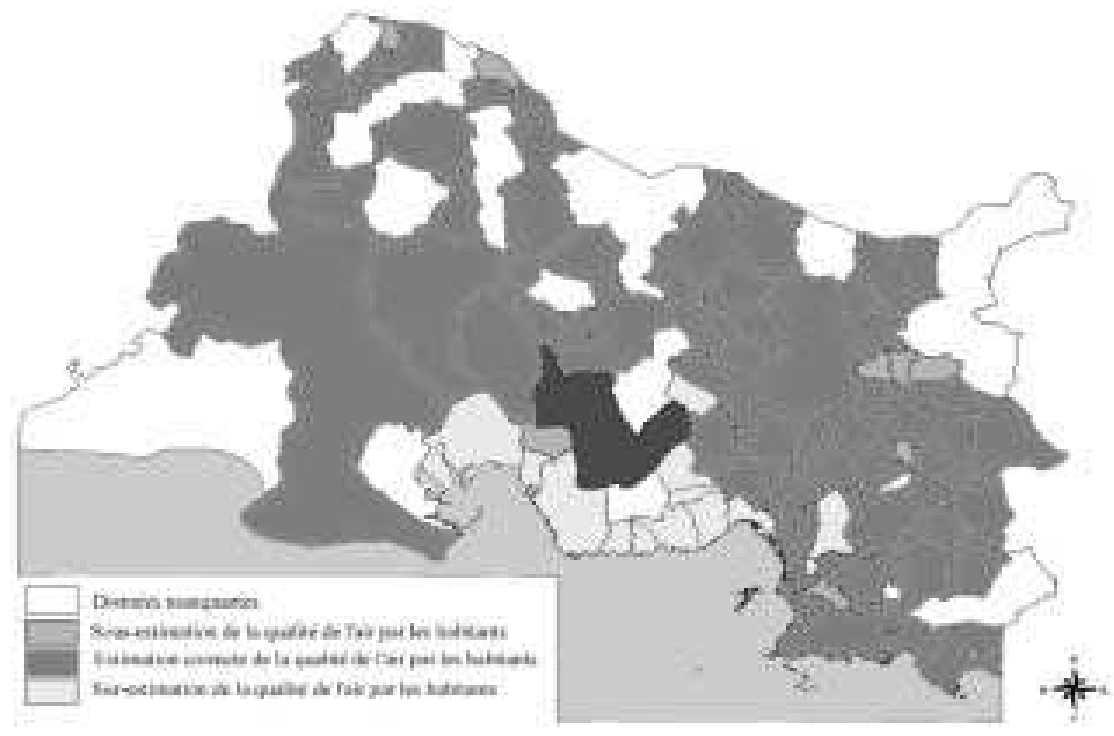

* Les données manquantes indiquent une absence de répondants dans cette commune.

Peut-on déduire de cette comparaison entre mesures techniques et perceptions individuelles, une erreur de perception moyenne des habitants des Bouches-du-Rhône ? Ne peut-on pas penser que les mesures des capteurs se trompent et que ce sont les sens humains qui détiennent «la » vérité de la pollution de l'air? Les entretiens effectués auprès des professionnels de la pollution de l'air ont montré que les décalages ou écarts entre mesures techniques et perceptions sensorielles, ne permettent pas pour autant d'inférer que les perceptions se trompent. Nous pouvons seulement constater que, en moyenne, par rapport aux mesures techniques, $20 \%$ des individus «sur-estiment » la qualité de l'air de leur domicile par rapport à la ville de Marseille, $20 \%$ la «sousestiment», et $60 \%$ l'estiment comme les appareils de mesure. La carte de la figure 2 présente l'écart entre la qualité de l'air mesurée techniquement et la qualité moyenne perçue par les personnes enquêtées ${ }^{1}$. Cette carte suggère une corrélation spatiale dans les erreurs de jugement de la pollution. En effet, on constate que les habitants soumis aux retombées de Fos (Fos, Martigues, l'ensemble du littoral jusqu'au $16^{\mathrm{e}}$ arrondissement de Marseille), de Berre (Rognac), et les habitants du $13^{\mathrm{e}}$ arrondissement de Marseille estiment que la qualité atmosphérique de leur lieu de résidence est meilleure que ce que traduisent les appareils de mesure. Le reste de la population enquêtée (soit $60 \%$ ) l'estime de la même manière, hormis les habitants du $10^{\mathrm{e}}$ arrondissement de Marseille, des petites communes de l'est de l'agglomération aixoise et de Saint-Mitre-les-Remparts qui la «sous-estiment».

Ces variables de perception et d'écart de perceptions des niveaux de pollution atmosphérique, calculées pour caque individu, sont utilisées dans l'analyse économétrique comme facteurs explicatifs des CAP.

1. La qualité moyenne est obtenue à partir de la question portant sur la perception de la pollution de l'air dans le lieu de résidence comparé à Marseille, recodée en cinq classes et agrégée sur l'ensemble des personnes habitant la commune. 


\section{RÉSULTATS ÉCONOMÉTRIQUES}

Tableau 1. Modèles estimés*

\begin{tabular}{|c|c|c|c|}
\hline \multirow{2}{*}{ Variables } & $\begin{array}{l}\text { CAP } \\
\text { sans informa- } \\
\text { tion }\end{array}$ & $\begin{array}{l}\text { CAP } \\
\text { avec informa- } \\
\text { tion }\end{array}$ & $\begin{array}{c}\text { CAP } \\
\text { mortalité }\end{array}$ \\
\hline & \multicolumn{3}{|c|}{$\begin{array}{c}\text { Paramètre } \\
\text { (p-value) }\end{array}$} \\
\hline Constante Hôtel de région & $\begin{array}{c}-27.647111 \\
(0.309933)\end{array}$ & $\begin{array}{c}-20.051725 \\
(0.441345)\end{array}$ & $\begin{array}{c}-39.908233 \\
(0.137928)\end{array}$ \\
\hline Constante Enq. téléphonique & $\begin{array}{c}-73.729427 \\
(0074993)\end{array}$ & $\begin{array}{c}-47.446303 \\
(0.205494)\end{array}$ & $\begin{array}{c}-68.267942 \\
(0.087334)\end{array}$ \\
\hline Âge & $\begin{array}{l}2.932692 \\
(0.082792)\end{array}$ & $\begin{array}{l}2.737218 \\
(0.091931)\end{array}$ & $\begin{array}{l}3.329839 \\
(0.054895)\end{array}$ \\
\hline Âge au carré & $\begin{array}{c}-0.046854 \\
(0.029856)\end{array}$ & $\begin{array}{l}-0.42719 \\
(0.035694)\end{array}$ & $\begin{array}{c}-0.044235 \\
(0.038378)\end{array}$ \\
\hline Niveau bac & $\begin{array}{c}24.645503 \\
(0.038583)\end{array}$ & $\begin{array}{c}24.394450 \\
(0.035249)\end{array}$ & $\begin{array}{c}18.107147 \\
(0.08397)\end{array}$ \\
\hline Études supérieures & $\begin{array}{c}28.546219 \\
(0.016813)\end{array}$ & $\begin{array}{c}26.392709 \\
(0.019280)\end{array}$ & $\begin{array}{c}20.623366 \\
(0.047120)\end{array}$ \\
\hline En activité professionnelle & $\begin{array}{r}22.151425 \\
(0.076953)\end{array}$ & $\begin{array}{l}17.797448 \\
(0.128841)\end{array}$ & $\begin{array}{l}8.118024 \\
(0.397190)\end{array}$ \\
\hline Au chômage & $\begin{array}{c}25.940670 \\
(0.051025)\end{array}$ & $\begin{array}{c}25.795041 \\
(0.047212)\end{array}$ & $\begin{array}{l}7.868501 \\
(0.433869)\end{array}$ \\
\hline Inactif & $\begin{array}{c}28.445892 \\
(0.112329)\end{array}$ & $\begin{array}{c}28.978713 \\
(0.103972)\end{array}$ & $\begin{array}{l}31.939485 \\
(0.078532)\end{array}$ \\
\hline Qualité de 1'air perçue à Marseille : bonne & $\begin{array}{c}-14.813976 \\
(0065541)\end{array}$ & $\begin{array}{c}-17.318035 \\
(0.036904)\end{array}$ & $\begin{array}{c}-13.712465 \\
(0.068825)\end{array}$ \\
\hline $\begin{array}{l}\text { Avoir une bonne connaissance de la pollu- } \\
\text { tion de l'air }\end{array}$ & $\begin{array}{l}18.532020 \\
(0.040428)\end{array}$ & $\begin{array}{l}17.086265 \\
(0.047000)\end{array}$ & - \\
\hline $\begin{array}{l}\text { Avoir changé ses habitudes à cause de la } \\
\text { pollution }\end{array}$ & $\begin{array}{l}15.796291 \\
(0.052657)\end{array}$ & $\begin{array}{l}13.318246 \\
(0.080615)\end{array}$ & - \\
\hline $\begin{array}{l}\text { Appartenir à une association environnemen- } \\
\text { tale }\end{array}$ & $\begin{array}{l}-62.844977 \\
(0.022723)\end{array}$ & $\begin{array}{c}-64.361401 \\
(0.017714)\end{array}$ & - \\
\hline Être non fumeur & $\begin{array}{l}17.20650 \\
(0.035801)\end{array}$ & $\begin{array}{l}19.246709 \\
(0.022656)\end{array}$ & $\begin{array}{l}15.031414 \\
(0.052855)\end{array}$ \\
\hline Paramètre $\hat{\alpha}$ (élasticité du CAP/revenu) & $\begin{array}{l}0.173947 \\
(<0.00001)\end{array}$ & $\begin{array}{l}0.186344 \\
(<0.00001)\end{array}$ & $\begin{array}{l}0.217133 \\
(<0.00001)\end{array}$ \\
\hline Offre de départ de l'enquête téléphonique & $\begin{array}{l}0.224674 \\
(0.011624)\end{array}$ & $\begin{array}{l}0.152405 \\
(0.039750)\end{array}$ & $\begin{array}{l}0.128408 \\
(0.072366)\end{array}$ \\
\hline Écart type Enq. téléphonique & $\begin{array}{l}64.717847 \\
(<0.00001)\end{array}$ & $\begin{array}{l}62.492778 \\
(<0.00001)\end{array}$ & $\begin{array}{l}53.974354 \\
(<0.00001)\end{array}$ \\
\hline Écart type Hôtel de région & $\begin{array}{l}123.98473 \\
(<0.00001)\end{array}$ & $\begin{array}{l}130.20655 \\
(<0.00001)\end{array}$ & $\begin{array}{l}101.17372 \\
(<0.00001)\end{array}$ \\
\hline $\begin{array}{l}\text { CAP moyen } \\
\text { Dispersion }\end{array}$ & $\begin{array}{l}427,03 \mathrm{~F} \\
223,98 \mathrm{~F}\end{array}$ & $\begin{array}{l}457,24 \mathrm{~F} \\
217,65 \mathrm{~F}\end{array}$ & $\begin{array}{l}405,36 \mathrm{~F} \\
210,06 \mathrm{~F}\end{array}$ \\
\hline $\begin{array}{l}\hat{\ell} \\
\text { Test LR de significativité jointe des variables } \\
\text { explicatives }\end{array}$ & $\begin{array}{c}-5872.07 \\
100.764 \\
(<0.00001)\end{array}$ & $\begin{array}{l}-5961.52 \\
82.08 \\
(<0.00001)\end{array}$ & $\begin{array}{c}4547.38 \\
44.08 \\
(<0.00001) \backslash\end{array}$ \\
\hline Taille de l'échantillon & 978 & 978 & 759 \\
\hline
\end{tabular}




\section{CALCUl D’une VED À PARTIR D'UN MODÈLE D'UTILITÉ AVEC DURÉE DE VIE ALÉATOIRE}

Considérons que les individus cherchent à maximiser la valeur espérée d'une fonction d'utilité indirecte sur l'ensemble de leur durée de vie restante. La fonction d'utilité à la date $t$, notée $u_{t}($.$) , est supposée croissante, strictement concave, deux fois continûment$ différenciable, additive et séparable dans le temps.

Notons $\mu_{j}$ le taux de mortalité d'un individu d'âge $j$. La fonction de survie à l'âge $j$ dépend des taux de mortalité à chaque âge de la façon suivante :

$$
\mathrm{S}_{j}=e^{-\int_{0}^{j} \mu(s) \mathrm{d} s}
$$

La probabilité d'être vivant à l'âge $t$ conditionnellement au fait d'avoir survécu jusqu'à l'âge $j, \mathrm{~S}_{t} / \mathrm{S}_{j}$, est notée $\mathrm{S}_{t, j}$. L'individu détermine son plan de consommation optimal $c_{t}$ sur son espérance de vie attendue selon le programme d'optimisation suivant :

$$
\operatorname{Max}_{c_{t}} \quad \mathrm{E}\left[u\left(c_{j}\right)\right]=\int_{j}^{\mathrm{T}} e^{-\delta(t-j)} \mathrm{S}_{t, j} u\left(c_{t}\right) \mathrm{d} t
$$

où $\mathrm{E}($.$) représente l'opérateur espérance ; T représente l'âge maximal que peut atteindre$ un être humain ; $c_{t}$ représente la consommation à l'âge $t$; et $\delta$ représente le taux individuel de préférence pour le présent, supposé indépendant de l'âge et du temps.

Dans le modèle usuel de cycle de vie et dans le cas de marchés parfait (voir Shepard et Zeckhauser [1984]), chaque individu est supposé choisir son plan de consommation intertemporel, compte tenu de sa richesse actuelle, de ses revenus futurs $y_{t}, t=j, \ldots, \mathrm{T}$ et des possibilités d'emprunts et de placements sur le marché des capitaux.

Ce modèle suppose l'existence d'un marché (parfait) des rentes viagères permettant le financement, par ceux qui décèdent, de la consommation de ceux qui survivent. Les individus peuvent alors choisir un plan de consommation satisfaisant une contrainte budgétaire sur la durée de vie espérée. Toutefois, même en l'absence d'un marché parfait, Blomqvist [2002] fait remarquer que, dans les pays développés, il existe des marchés privés d'assurance-vie et que l'État, par un système public de retraite ou un système d'assurance vieillesse, garantit un niveau minimal de consommation. De plus, dans de nombreux cas, la famille agit, au moins partiellement, comme un tel système par des transferts intergénérationnels (voir Kotlikoff et Spivak [1981]).

Ainsi, il est raisonnable que la contrainte budgétaire intertemporelle d'équilibre que doit vérifier chaque individu soit telle que :

$$
\int_{j}^{\mathrm{T}} e^{-r(t-j)} \mathrm{S}_{t, j}\left(y_{t}-c_{t}\right) \mathrm{d} t=0
$$

où $y_{t}$ est le revenu à l'âge $t, t \geqslant j$, et $r$ le taux d'intérêt d'un actif sans risque.

La maximisation de (11) sous contrainte de (12) conduit au chemin de consommation optimal $c_{t}^{*}$ de l'individu sur le restant de son cycle de vie (voir Blomqvist [2002]) et au Hamiltonien suivant :

$$
\mathrm{H}_{j}=\int_{j}^{\mathrm{T}} u\left(c_{t}^{*}\right) e^{-\delta(t-j)} \mathrm{S}_{t, j} \mathrm{~d} t+\lambda^{*} \int_{j}^{\mathrm{T}} e^{-r(t-j)} \mathrm{S}_{t, j}\left(y_{t}-c_{t}^{*}\right) \mathrm{d} t
$$

où $\lambda_{t}$ représente le multiplicateur de Lagrange et l'exposant ${ }^{*}$ les valeurs optimales. On peut alors définir la valeur d'évitement d'un décès à l'âge $j$, noté $\mathrm{VED}_{j}$, comme la contrepartie monétaire de l'utilité actualisée sur la durée de vie espérée (Blomqvist [2002]; Johansson [2001]), soit :

$$
\operatorname{VED}_{j}=\frac{1}{\lambda^{*} e^{-r j}} \int_{j}^{\mathrm{T}} \mathrm{S}_{t, j}\left[u\left(c_{t}^{*}\right) e^{-\delta(t-j)}+\lambda^{*} e^{-r(t-j)}\left(y_{t}-c_{t}^{*}\right)\right] \mathrm{d} t
$$


Dans cette équation, l'utilité est convertie en termes monétaires en divisant par $\lambda^{*}$, interprété comme l'utilité marginale de la monnaie.

Considérons maintenant une action qui, durant un court intervalle de temps D, modifierait de façon marginale le taux de mortalité à l'âge $j, \mu_{j}$, d'une grandeur $d \mu$, indépendante de l'âge. Ceci implique une variation $d \mathrm{~S}_{t, j}$ de la probabilité conditionnelle de survie d'un individu à l'âge $j$. Notons $\mathrm{CAP}_{j}$ le consentement à payer (CAP) immédiat d'un individu d'âge $j$ pour cette variation $d \mathrm{~S}_{t, j}$ de sa probabilité conditionnelle de survie à l'âge $j$. Il est alors possible de déterminer ce $\mathrm{CAP}_{j}$ qui maintiendrait l'utilité espérée constante, soit d'après (13) :

$\mathrm{H}_{j}^{*}=\int_{j}^{\mathrm{T}} u\left(c_{t}^{*}\right) e^{-\delta(t-j)} \mathrm{dS}_{t, j} \mathrm{~d} t+\lambda^{*} \int_{j}^{\mathrm{T}} e^{-r(t-j)} \mathrm{dS}_{t, j}\left(y_{t}-c_{t}^{*}\right) \mathrm{d} t-\lambda^{*} e^{-r j} \mathrm{CAP}_{j}=0$

On considère alors que l'individu paye le montant $\mathrm{CAP}_{\tilde{\tilde{S}}}$ pour diminuer son taux de mortalité pendant l'intervalle de temps D. Soit $\mathrm{S}_{t, j}\left(\operatorname{resp} . \tilde{\mathrm{S}}_{t, j}\right)$ la fonction de survie avant (resp. après) la modification du taux de mortalité $d \mu$. $\widehat{S}_{t, j}$ s'écrit de la manière suivante :

$$
\tilde{\mathrm{S}}_{t, j}=e^{-\int_{j}^{j+\mathrm{D}}(\mu(s)-\mathrm{d} \mu) \mathrm{d} s} e^{-\int_{j+\mathrm{D}}^{t} \mu(s) \mathrm{d} s}=e^{\mathrm{D} d \mu} \mathrm{S}_{t, j}
$$

Nous avons donc :

$$
d \mathrm{~S}_{t, j}=\tilde{\mathrm{S}}_{t, j}-\mathrm{S}_{t, j}=\mathrm{S}_{t, j}\left[e^{\mathrm{D} d \mu}-1\right] \approx \mathrm{S}_{t, j} \mathrm{D} d \mu \quad \text { pour } \quad \mathrm{D} d \mu \text { petit. }
$$

En combinant les équations (14) et (15), on réécrit $\mathrm{VED}_{j}$ comme :

$$
\begin{aligned}
\operatorname{VED}_{j} & =\frac{1}{\lambda^{*} e^{-r j}} \int_{j}^{\mathrm{T}} \mathrm{S}_{t, j}\left[u\left(c_{t}^{*}\right) e^{-\delta(t-j)}+\lambda^{*} e^{-r(t-j)}\left(y_{t}-c_{t}^{*}\right)\right] \mathrm{d} t \\
& =(\mathrm{D} d \mu)^{-1} \mathrm{CAP}_{j}
\end{aligned}
$$

\section{E. CALCUl DE LA VARIATION DE LA PROBABILITÉ DE DÉCÈS $d \mu$}

Une fonction de Gompertz du type $a e^{b t}$ est généralement utilisée (Leksell et Rabl [2001]) pour modéliser la probabilité de décès à l'âge $t, \mu_{t}$ pour son excellente adéquation tant pour l'espèce humaine que pour la plupart des espèces animales. La fonction suivante est estimée sur données françaises (sources, INSEE [1997]) :

$$
\begin{aligned}
\mu_{t} & =a e^{b t}=0,00007346 e^{0,081245106 t}, \\
\mathrm{R}^{2} & =0,995910712
\end{aligned}
$$

La fonction de survie conditionnelle correspondante s'écrit alors comme :

$$
\mathrm{S}_{t, j}=e^{-\int_{j}^{t} \mu(s) \mathrm{d} s}=e^{-\int_{j}^{t} a e^{b s} \mathrm{~d} s}
$$

L'effet de l'exposition à long terme à la polution atmosphérique sur la mortalité s'exprime généralement sous la forme d'un risque relatif (RR), et suppose que le taux de mortalité $\mu_{t}$ est affecté de façon proportionnelle par l'exposition à la pollution atmosphérique $^{1}$ :

$$
\mu_{t}=\mathrm{RR} \mu_{t}^{0}
$$

où $\mu_{t}^{0}$ est le taux de mortalité qui prévaudrait dans la population en l'absence de pollution atmosphérique.

1. Les études de cohortes ne sont pas encore suffisamment nombreuses pour permettre d'obtenir des RR dépendant de l'âge $t$. La variation de la probabilité de décès $d \mu$ sera donc constante pour tout âge. 
Il est donc possible de déterminer la variation $d \mu$ de la probabilité de décès correspondant à l'application du risque relatif associé à l'exposition entre l'âge médian (35 ans en France, INSEE [1997]) et 80 ans. On obtient $d \mu$ en résolvant l'équation suivante :

$$
e^{-\mathrm{RR}^{-1} \int_{35}^{80} a e^{b s} \mathrm{~d} s}=e^{-\int_{35}^{80}\left(a e^{b s}-\mathrm{d} \mu\right) \mathrm{d} s}
$$

Soit,

$$
d \mu=\frac{\int_{35}^{80} a e^{b s} \mathrm{~d} s\left[1-\mathrm{RR}^{-1}\right]}{45}
$$

Dans la mesure où la pollution atmosphérique est un cocktail de multiples gaz et de substances en suspension il n'en existe pas de mesure unique. Le nombre de jours de pollution est utilisé dans le scénario pour expliciter dans le langage courant la notion de pollution (le niveau de l'indice Atmo, les éventuels dépassements de seuils et les restrictions de circulation associées sont par exemple comptabilisés par journée). Pour le calcul d'un RR associé à la mortalité, il convient de retenir un unique polluant, puisque agréger des effets associés à divers polluants conduirait à une surestimation du fait de la corrélation résultant de sources d'émission communes. Les particules en suspension d'un diamètre inférieur à $10 \mu \mathrm{m}$ (PM10) sont donc retenues, car elles représentent un bon indicateur des effets sanitaires de la pollution atmosphérique globale hormis la pollution oxydante et que les données épidémiologiques associées sont nombreuses.

L'exposition moyenne pondérée de la population française à ce polluant est évaluée par modélisation à $23,5 \mu \mathrm{g} / \mathrm{m}^{3}$ par Filliger et al. [1999], cohérente avec les réseaux de mesure Airmaraix (partie la plus urbanisée du département) avec $28 \mu \mathrm{g} / \mathrm{m}^{3}$, et Airfobep (partie plus industrielle et rurale) avec $21 \mu \mathrm{g} / \mathrm{m}^{3}$. Le seuil de $7,5 \mu \mathrm{g} / \mathrm{m}^{3}$ est retenu comme le niveau naturel qui prévaudrait en l'absence d'émissions anthropiques.

Suivant Künzli et al. [2000], le risque relatif de mortalité relatif à l'exposition, est évalué à 1,043 pour une variation de $10 \mu \mathrm{g} / \mathrm{m}^{3}$ du niveau des PM10, soit $\mathrm{RR}=1+(0,0043 \Delta c)$ pour une variation de $\Delta c \mu \mathrm{g} / \mathrm{m}^{3}$. Les trois scénarios correspondent à $\Delta c=16 \mu \mathrm{g} / \mathrm{m}^{3}$ pour une suppression totale, $\Delta c=8 \mu \mathrm{g} / \mathrm{m}^{3}$ pour une suppression de moitié, et $\Delta c=4 \mu \mathrm{g} / \mathrm{m}^{3}$ pour une suppression d'un quart.

La formule (23) permet alors de calculer l'impact de la variation du niveau de pollution atmosphérique sur la probabilité de décès, $d \mu$. Pour une réduction de moitié, $d \mu^{8}=0,0004328$, pour une réduction totale, $d \mu^{16}=0,0008378$ et pour une réduction d'un quart, $d \mu^{4}=0,00022^{1}$. Le membre droit de l'équation (22) conduit ensuite à l'expression en termes de décès supplémentaires utilisée dans le scénario. La probabilité de survie conditionnelle $S_{35,80}$ est égale à 0,5568 pour $d \mu=0$ (avant réduction), à 0,5677 pour $d \mu^{8}$ et à 0,5781 pour $d \mu^{16}$ soit, ramenée à 100 personnes, à 1,09 personne (arrondi à 1) pour une réduction de moitié et à 2,13 personnes (arrondi à deux) pour une réduction totale. 